\title{
Research Article \\ Smart Cylindrical Dome Antenna Based on Active Frequency Selective Surface
}

\author{
Tongyu Ding, ${ }^{1}$ Shaoqing Zhang, ${ }^{2}$ Liang Zhang, ${ }^{1}$ and Yanhui Liu ${ }^{3}$ \\ ${ }^{1}$ College of Information Engineering, Jimei University, Xiamen 361021, China \\ ${ }^{2}$ Aviation Key Laboratory of Science and Technology on Electromagnetic Environmental Effects, \\ Shenyang Aircraft Design and Research Institute, Shenyang 110035, China \\ ${ }^{3}$ Department of Electronic Science, Xiamen University, Fujian 361005, China \\ Correspondence should be addressed to Liang Zhang; liangzhang@jmu.edu.cn
}

Received 8 July 2016; Revised 8 December 2016; Accepted 27 December 2016; Published 7 February 2017

Academic Editor: Diego Caratelli

Copyright (C) 2017 Tongyu Ding et al. This is an open access article distributed under the Creative Commons Attribution License, which permits unrestricted use, distribution, and reproduction in any medium, provided the original work is properly cited.

\begin{abstract}
In this paper, we proposed a beamforming antenna, which is realized using an omnidirectional antenna in the center surrounded by a cylindrical smart dome. The smart dome is made of 16 active frequency selective surface columns of which the amplitude and phase response can be continuously tuned by varying the bias voltages of the employed varactors. Thus, the performance of the proposed antenna could achieve higher gain, better nulling level, and more agility than many switch methods-based cylindrical reconfigurable antennas. Moreover, in order to overcome the unavailable analytical synthesis caused by complex mutual coupling between columns, we develop a genetic algorithm based optimization system and conducted a serial of experiments to evaluate the high-gain, nulling, continuously steering, and frequency-invariant ability. The results show that, during the frequency tunable range of the AFSS $(2.0 \mathrm{GHz}$ to $2.7 \mathrm{GHz})$, the antenna can offer an additional gain of up to $6.57 \mathrm{~dB}$ and nulling level of $-56.41 \mathrm{dBi}$. For the high-gain modes, the $-3 \mathrm{~dB}$ beam widths are $26^{\circ}-34^{\circ}$, which offers enhanced angular resolution compared with other reported beam-sweeping work. Furthermore, the radiation pattern is continuously steerable.
\end{abstract}

\section{Introduction}

With the development of wireless industry, adaptive and smart antenna systems are developed to solve the interference problems $[1,2]$. Analog phased array and multiple-channel digital signal process can be used to build smart antenna systems $[3,4]$. However, both of these methods are complicated and expensive. Electronically steerable antennas based on active frequency selective surface (AFSS) are studied in recent years to provide economical beam steering solutions. Both planar and cylindrical conformal arrays are reported [5-9]. The cylindrical ones have similar configurations; they are mostly constructed by a central omnidirectional antenna and several surrounding AFSS columns (with the shape of a cylinder). These designs offer low cost and highly agile solutions with the purpose of radiation pattern manipulation. The majority of these AFSSes are based on PIN diodes. There are only two states for PIN diodes, on and off. Thus, the idea of operating this kind of antenna is turning some of the AFSS columns reflective, while turning the others transparent. In this way, nearly half of the radiation energy is reflected and a better additional gain is achieved. The $3 \mathrm{~dB}$ beam widths are about $70^{\circ}$ in the azimuth plane [6-8]. A more recent research showed that the beam-width of this kind of antennas can be narrowed to $47^{\circ}$ with an optimized dimension [10]. These on/off methods-based antennas are all sector antennas which means that they are not continuously steerable. In the previous work [9], varactors based AFSSes were used to achieve continuously steerable ability. However, amplitude and phase responses of the AFSSes were not considered in that work.

As reported in [11], manipulation of the radiation pattern of a horn antenna by using the amplitude and phase response of an AFSS proved to be available. Inspired by this work, for the cylindrical cases, if the amplitude and phase response can be controlled continuously, they are quite similar to conformal weighted and phased arrays. In such cases, higher gain, deeper nulling level, and better agility can be expected as 


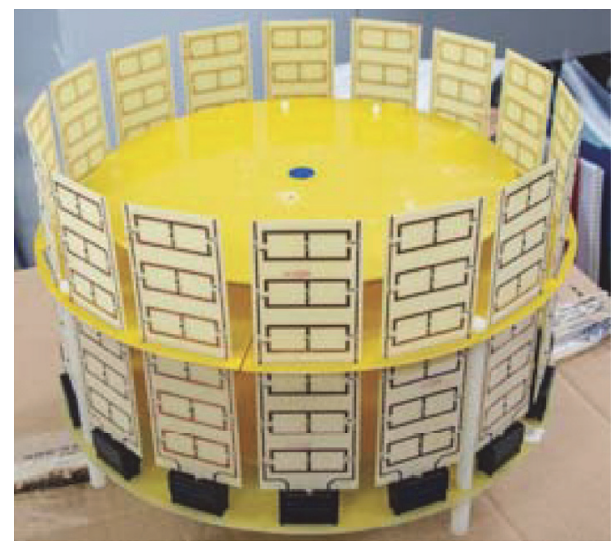

(a)

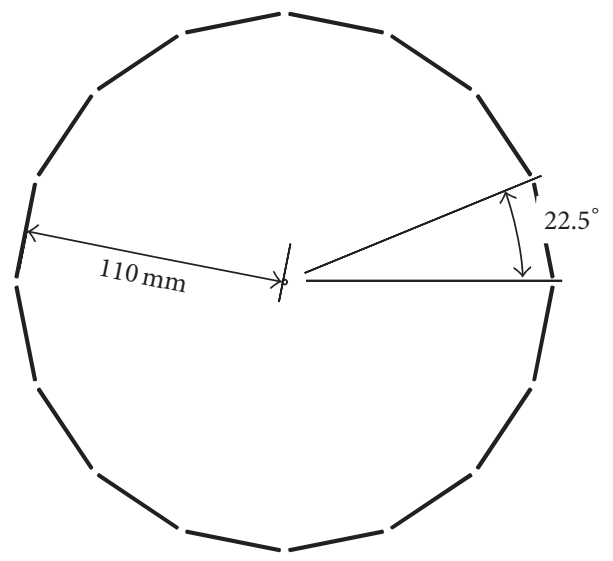

(c)

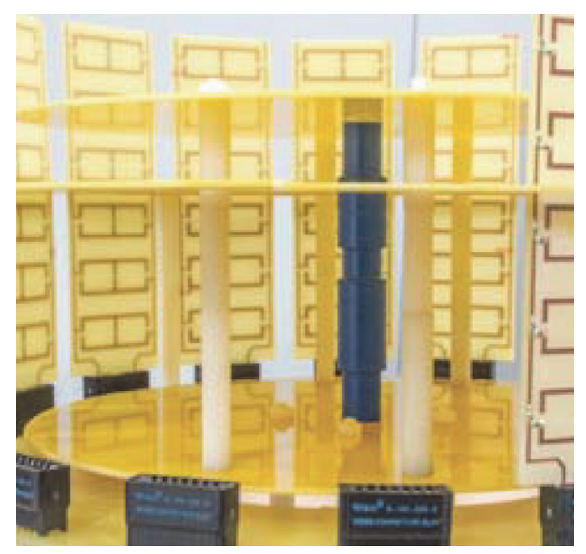

(b)

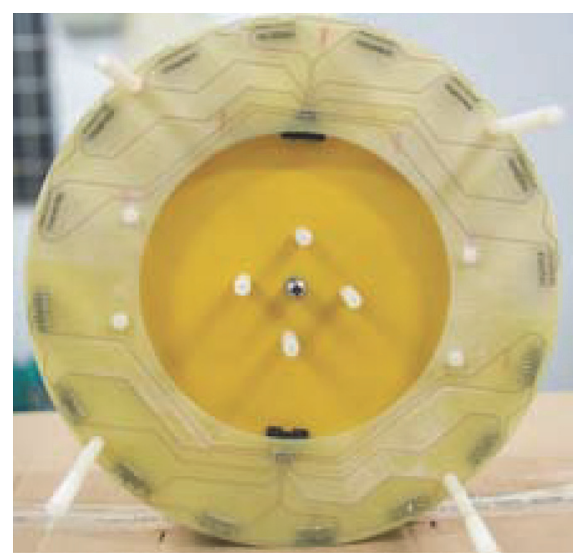

(d)

Figure 1: Antenna structure. (a) Photo of the antenna. (b) Central dipole antenna. (c) Cross section and dimensions. (d) Bottom plane.

a smart antenna [12]. For this purpose, a cylindrical AFSS based antenna was developed in this work. It takes advantage of the phase tuning ability of the AFSS to realize a higher additional gain and deeper nulling level. A self-contained optimization algorithm, which is capable of effectively enhancing the performance of the antenna, is also developed to realize the optimization. The radius of this prototype is enlarged to $1.76 \lambda$ of the central frequency. Moreover, since this antenna has an essential different working mechanism compared with the reported ones $[5-8,10]$, which used PIN-diode for the AFSSes and can only switch between the on and off modes, we would prefer to reconsider it as a smart dome and an inner emitter. The emitter is a general omnidirectional antenna and it can be replaced by many different models with vertical polarization direction, while the radiation pattern manipulation ability is realized by the dome.

The rest of this paper is organized as follows. In Section 2, the structure of this antenna and the design of the AFSS are introduced. The amplitude and phase responses of the AFSS are discussed. The complexity of performing beamforming on this antenna is explained. In Section 3, an artificial intelligence (AI) based optimizing system is built to overcome the above-mentioned problems and evaluate the performance of the antenna. We use genetic algorithm (GA) method to optimize the antenna for different purposes. The performances of the gain, nulling level, frequency-invariant beam, and agility are revealed. Finally, the paper is concluded in Section 4.

\section{Design of the Antenna}

2.1. Structure of the Antenna. The antenna is constructed by a dipole antenna in the center and 16 AFSS columns surrounding it, as shown in Figure 1 (the number of AFSS columns is currently determined by the number of DA channels in the self-made voltage controller). The dipole acts as a radiator emitting omnidirectional vertically polarized signal. The bottom plane is an annular shaped, single-layer FR4 PCB with 16 golden-finger jackets and two multiple pin connectors. A pair of golden fingers is added to the bottom of each AFSS column so it can be plugged into the jacket, where the bias voltage is added. There are 6 unit cells within each column. The diameter of the antenna is $220 \mathrm{~mm}$ and the height is $133 \mathrm{~mm}$. Dielectric FR4 board, nylon sticks, and screws are added to help holding the dipole antenna and AFSS columns in position.

The mechanism of this antenna is illustrated in Figure 2. The signal is emitted by the central omnidirectional dipole 


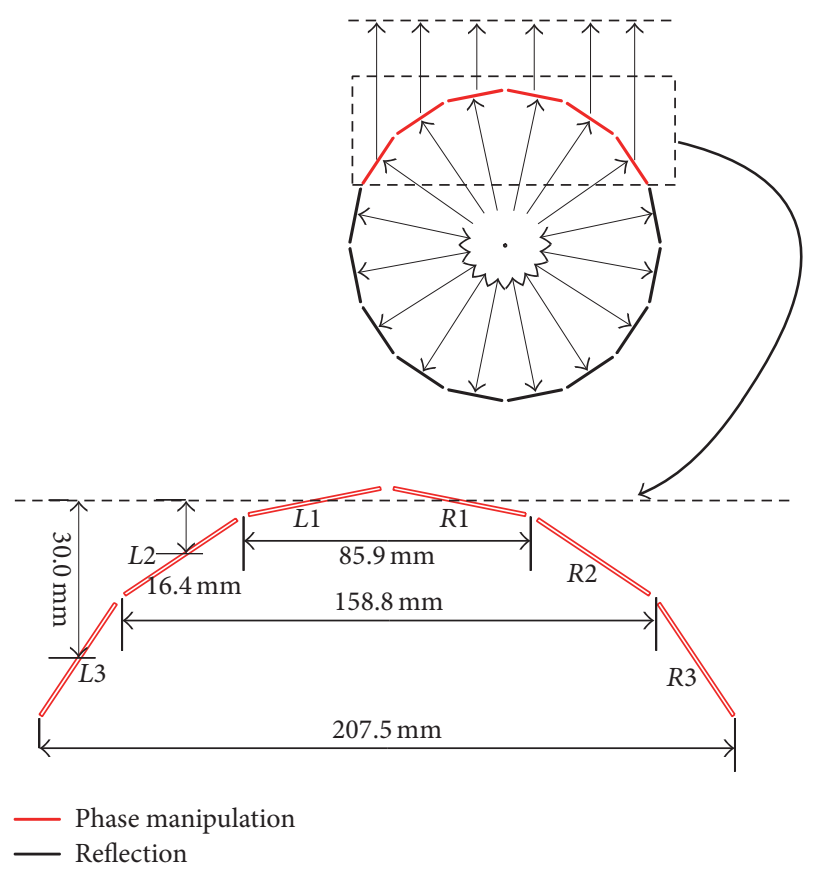

FIGURE 2: Illustration of the theory.

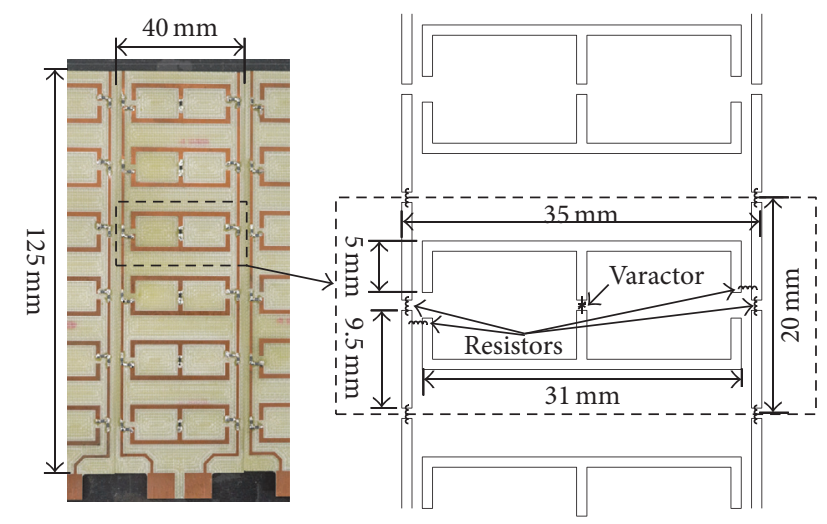

FIGURE 3: AFSS structure and dimensions.

and reflected on the AFSS columns, which are tuned into reflective status at the working frequency. The other AFSS columns (for this demonstration, there are six) work at different states between transparent and reflective modes to manipulate the phase. As shown in Figure 2, there are $16.4 \mathrm{~mm}$ and $30.0 \mathrm{~mm}$ distance differences. For different wavelength, they correspond to different phase differences.

2.2. Design, Measurement and Discussion of the AFSS. The structure and dimensions of the developed AFSS are shown in Figure 3. The AFSS patch is constructed by two mirrored $E$ like patches. The original design of this patch can be traced back to [13], and it is also known as the electric-LC (ELC) resonator. It is later successfully modified into an AFSS with broadband tunability [14]. In this work, the unit cells of the AFSS were fabricated on a single-layer FR4 board (thus no via holes or patches on the other side) and were rotated by $90^{\circ}$ to manipulate the vertically polarized signal. We used 16 such AFSS columns, each applied with an independently controlled bias voltage, to form the designed smart dome. The bias voltages powered to each column were controlled by a self-contained artificial intelligence program, optimizing the combinations of bias voltages to an optimum status, thus making the AFSS behavior outperform those in $[13,14] .10 \mathrm{~K}$ ohm resistors were added on the bias grid for the purpose of isolating the unit cells by choking the high frequency current between unit cells. The varactors used in this work are BB857, manufactured by Infineon. It is a surface-mounted model with SCD80 package. It is broadband tunable with a variable capacitance $C_{T}$ from $0.54 \mathrm{pF}$ to $6.6 \mathrm{pF}$, a low series resistance of $r_{S}=1.5 \mathrm{ohm}$, and the series inductance $L_{S}=$ $0.6 \mathrm{nH}$. When the varactor is reversely biased, the maximum current is $200 \mathrm{nA}\left(V_{R}=30 \mathrm{~V}\right)$, implying that the minimum resistance is $150 \mathrm{M}$-ohm, it is much larger than the $10 \mathrm{~K}$-ohm resistors. Thus, the DC bias voltage drop caused by the choke resistors is negligible. For the same purpose, two horizontally placed resistors were added to connect the bias grid and the mirrored $E$ patches.

The amplitude and phase responses under different voltages were measured in a microwave anechoic chamber. As shown in Figure 4, an isolation screen was built using steel plate with an aperture in the center. RF anechoic material was glued on both sides of the screen. Two horn antennas were set up pointing to each other through the aperture. $S_{21}$ between these two horn antennas were measured when FSS columns were installed covering the aperture with a distance of $45 \mathrm{~mm}$ to each other. The $S_{21}$ difference between this setup and the open aperture setup is the transmission coefficient of the FSS. This method is already widely used such as in [15-18]. The measured FSS screen was constructed by 6 FSS columns and the bias voltage was added at the bottom. The dimensions in Figure 4 are as follows: $L_{1}=1 \mathrm{~m}, L_{2}=1.5 \mathrm{~m}, H_{1}=W_{1}=2 \mathrm{~m}$, $W_{2}=30 \mathrm{~cm}$, and $H_{2}=20 \mathrm{~cm}$. Since the aim of this preliminary experiment is mainly to validate the effectiveness of the designed prototype and its capability of frequency tuning, we choose these setup dimensions according to the experimental environment, which is a $3 \mathrm{~m} \times 3 \mathrm{~m}$ anechoic chamber, due to the reason of economy, and so on (theoretically, the distance of the two antennas should be as large as possible to simulate the situation of far field plane wave).

Simulated and measured results are shown in Figure 5. The resonant frequency of this AFSS can be tuned from $2.0 \mathrm{GHz}$ to $2.75 \mathrm{GHz}$ with an insertion loss of more than $10 \mathrm{~dB}$. As the performance of materials in the experiment may decrease from the standard value set in the simulation due to aging problems, and so on, the experimental results are slightly deviated from simulation ones. As discussed in the previous work [9], the total working frequency range $\mathrm{BW}_{\text {working }}$ is no more than the sum of the frequency tunable range $\mathrm{BW}_{T}$ and stop-band frequency range $\mathrm{BW}_{R}(1)$. Thus the developed AFSS can be used for building electronically steerable antennas from $2.0 \mathrm{GHz}$ to $2.75 \mathrm{GHz}$, if an insertion loss of more than $10 \mathrm{~dB}$ is required. This frequency spectrum 


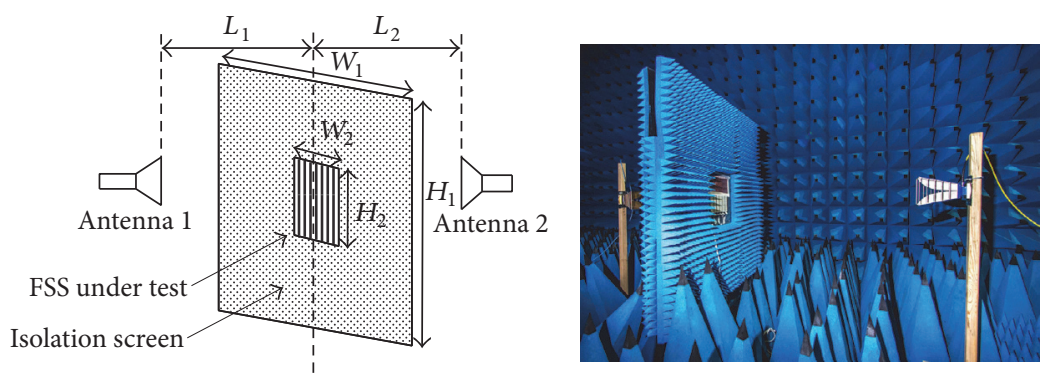

Figure 4: Measurement setup illustration and photo.

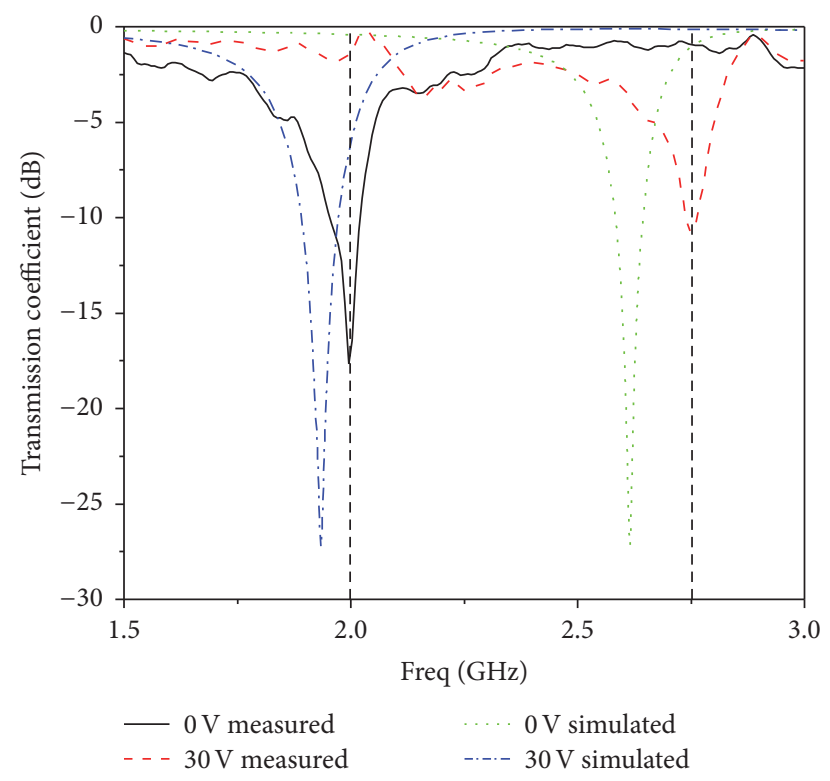

FIGURE 5: Simulated and measured transmission coefficients of the AFSS.

covers some part of WCDMA, some part of LTE, and $2.4 \mathrm{GHz}$ ISM band:

$$
\mathrm{BW}_{\text {working }} \leq \mathrm{BW}_{T}+\mathrm{BW}_{R} \text {. }
$$

Besides the tunable range, the amplitude and phase response at a given frequency point is directly related to beamforming. To measure such data, we altered the bias voltage from $0 \mathrm{~V}$ to $30 \mathrm{~V}$ with a step of $0.1 \mathrm{~V}$ and read the transmission coefficient and phase response versus voltage data at each $100 \mathrm{MHz}$ from the PNA 5227A using a Python program through LAN based VISA interface. Without loss of generality, the result at $2.4 \mathrm{GHz}$ is shown in Figure 6. It is not difficult to find out that the relationship between the bias voltage and the amplitude or the phase response is nonlinear. At different frequencies, the transmission coefficients and phase respond to different voltages, and the phase tunable range is also different.

For the purpose of beamforming, phase response is an important parameter. The measured phase responses for different frequency samples from $2.0 \mathrm{GHz}$ to $2.7 \mathrm{GHz}$ stepping $0.1 \mathrm{GHz}$ are shown in Figure 7 (simulations are not conducted

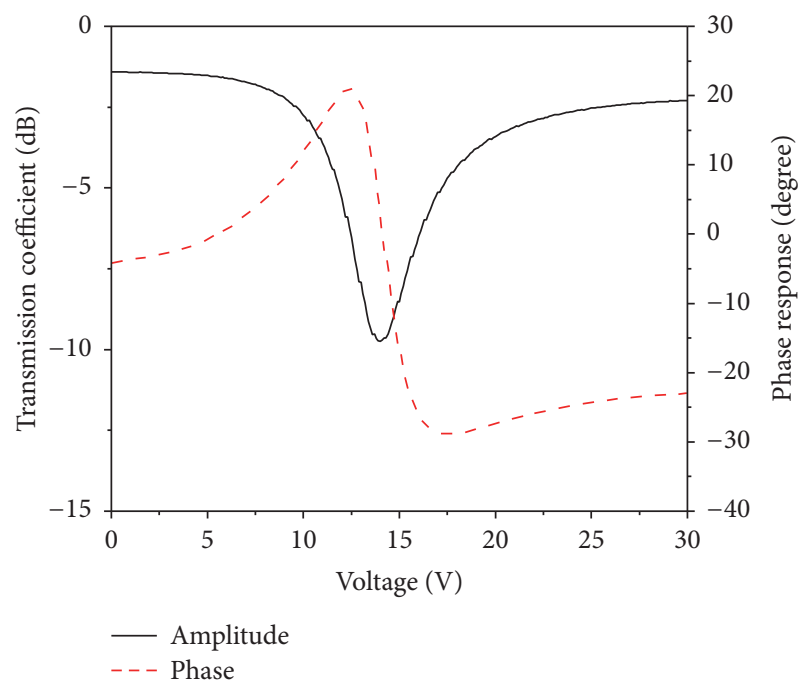

FIGURE 6: Measured results of transmission coefficient and phase response under different bias voltages at $2.4 \mathrm{GHz}$.

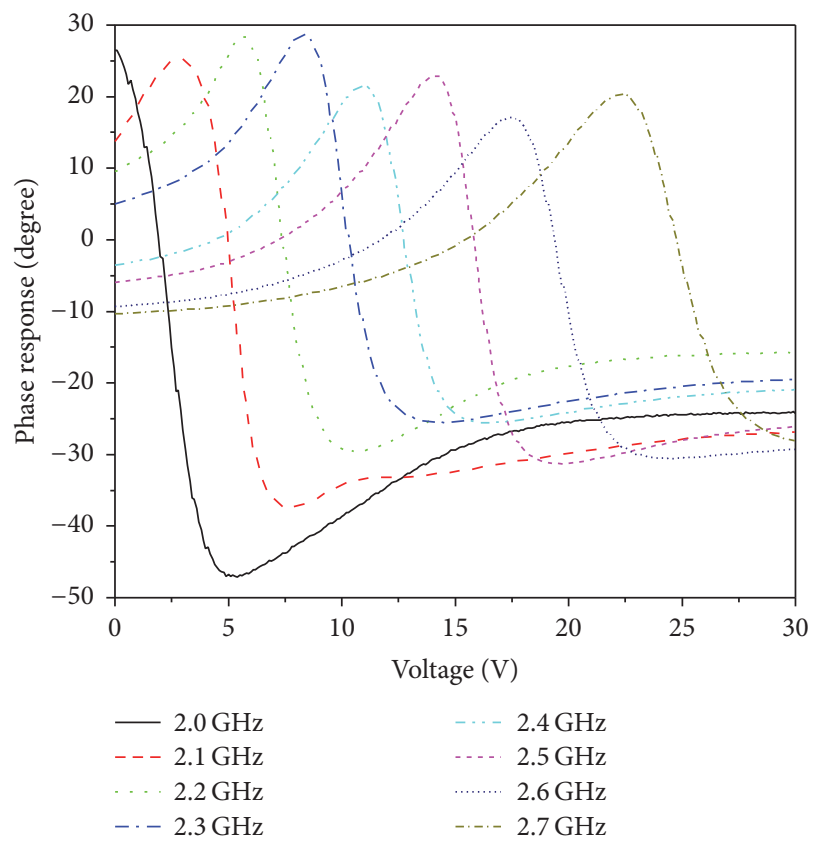

Figure 7: Measured phase response for different frequencies. 


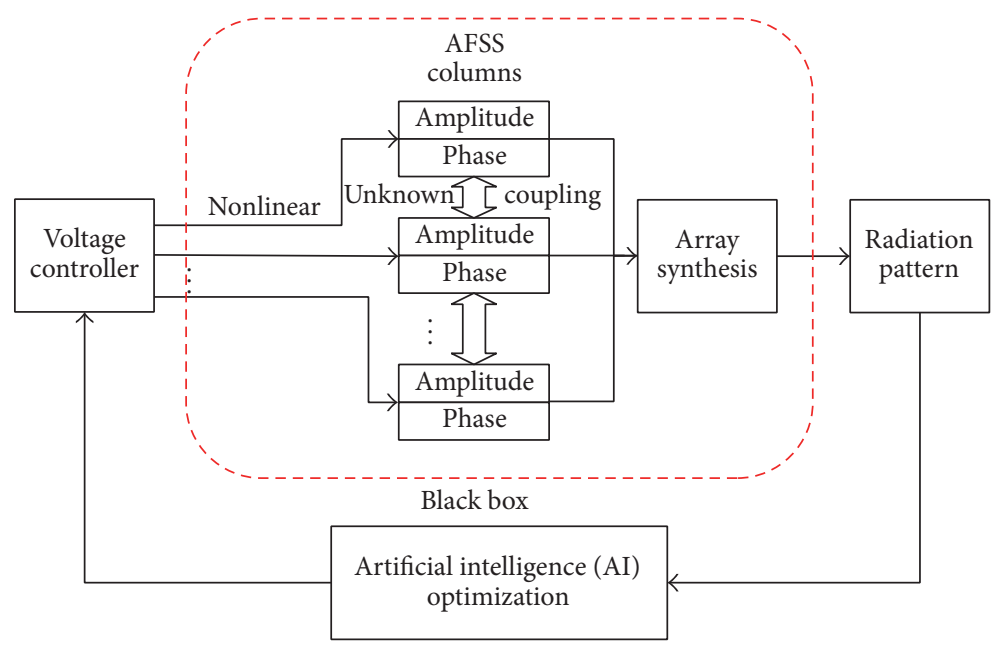

FIGURE 8: Measured phase response for different frequencies.

here for the reason of low efficiency and inaccuracy). The measured results show that the tunable phase response range is generally larger for the lower frequencies. At $2.1 \mathrm{GHz}$, the tunable range is $62.8^{\circ}$, while at $2.6 \mathrm{GHz}$ it is $47.6^{\circ}$. This behavior means that this AFSS has a better phase shifting ability within lower frequency band than the higher. For the fixed structure, the phase tuning requirement is given by physical length as shown in Figure 2. In this work the phase needs to be tuned to compensate $16.4 \mathrm{~mm}$ and $30 \mathrm{~mm}$. That is $43.5^{\circ}$ and $79.4^{\circ}$ for $2.1 \mathrm{GHz}$, while being $51.2^{\circ}$ and $93.6^{\circ}$ for $2.6 \mathrm{GHz}$. That means for the lower frequency band it is easier to satisfy the phase shifting requirement.

For the proposed antenna, the AFSS columns are very close to each other; thus the mutual coupling can be quite strong. Mutual coupling is usually considered when synthesizing a dense array. For a traditional array, multiple elements are fed though physically connected ports. It can be analyzed using a classical mutual impedance matrix method. This method is based on the voltage and current of the feed ports, as detailed in [19]. For the antenna in this work, these ports do not exist, and the interferences definitely happen before the signal reaches the AFSS columns. Thus, this method is not suitable for this case. An alternative approach is space-fed arrays [20-23]. However, to the best of the authors' knowledge, there is seldom discussion on the mutual coupling of these arrays.

Schneider and Munk proposed a method to calculate the scattering of super dense dipole array [24]. In this reference, the mutual coupling depends on the details of the unit cells, when the incident wave and the dipoles are not aligned. This method is based on the condition that all the elements are the same and uniformly distributed. This situation is very similar to our work. However, a big difference is that, in [24], all the elements are the same and static; in our work the AFSSes are supposed to be working under different voltages, which means that they have different states. This problem is much more complicated than the uniform cases. Also, Floquet mode is no longer suitable.
The flowchart of this problem and the idea to overcome it are shown in Figure 8. When 16-channel voltages are generated, the amplitudes and phases of the AFSS columns respond to the voltages. Radiation pattern can be synthesized if the amplitude and phase values are known. However, the relationship between the voltage and the states of the AFSS columns is nonlinear and the coupling between the columns is unknown. Furthermore, the amplitude and phase responses are not individual. Thus, the synthesis cannot be performed. To solve this problem, we considered the relationship between the voltages and the radiation pattern as a black box. An observer was added in the far field area to acquire the radiation level of the target direction. This radiation level is sent to an artificial intelligence (AI) based program and the relationship between the voltages and radiation pattern at the target direction is established. When a goal is assigned to the AI program, it can change the voltages to optimize the radiation pattern to reach the goal.

\section{Genetic Algorithm Based Experimental Investigation}

To realize the idea shown in Figure 8, we developed a genetic algorithm (GA) based program and a voltage controller. There are many other artificial intelligence methods. The reason we choose GA is because it is a global optimization method, and it is not complicated for programming.

3.1. The Genetic Algorithm Based Optimization System Setup. Artificial intelligence technologies have been well developed for decades. Studies on using GA to optimize arrays have been well reported [25-27], most of which used GA as a synthesis method to optimize the calculated radiation patterns. Different to these works, we developed the GA optimization system not only based on software but also on hardware. As can be seen from the block chart of Figure 9, the testing antenna and the developed antenna are both installed in a microwave anechoic chamber. Two coaxial 


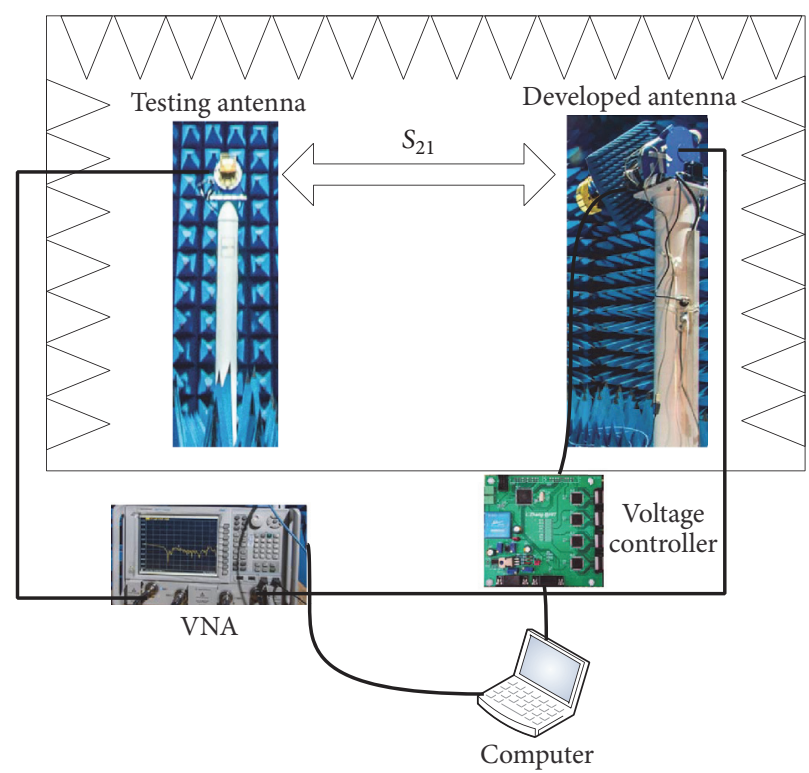

FIGURE 9: Illustration of the genetic algorithm based platform.

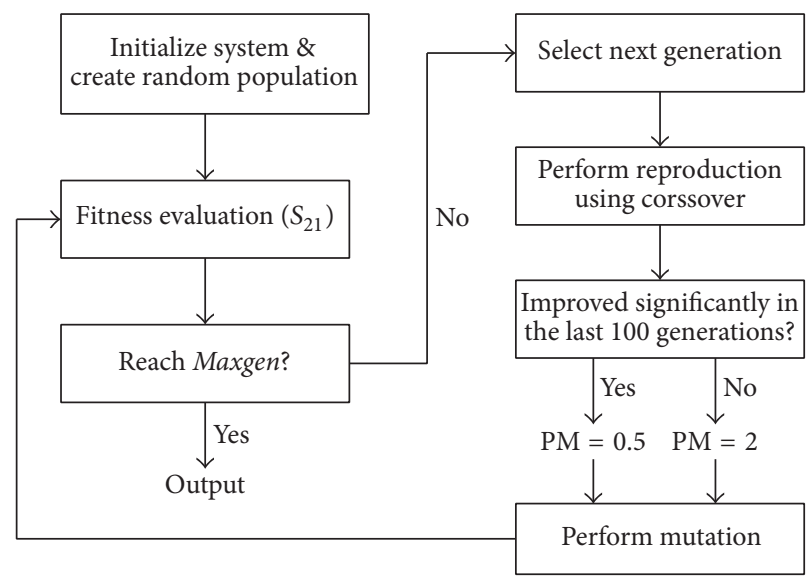

Figure 10: Program flowchart.

cables connect these two antennas and a N5227A vector network analyzer. When the measurement starts, the VNA is able to acquire the $S_{21}$ parameter between the two antennas. A self-made multichannel voltage controller connects the AFSS smart dome to provide several channels of bias voltages, and the GA-based program running on a computer controls the values of these bias voltages.

The working flow is as follows. The GA program randomly generates a 16-value combination, the range of which is limited within 0 to 30 . This combination is used as an individual in this GA program. Then the computer sends this combination command to the voltage controller to generate 16 voltage levels. The radiation pattern changes consequently. When the status is stable, the computer triggers the VNA to capture the $S_{21}$ amplitude and takes it back. The score for this combination is calculated based on this $S_{21}$ amplitude.
This 16-value combination is transformed to a binary code and used in the crossing-over and mutation procedure. For high-gain optimization, a higher score is given to a higher $S_{21}$ amplitude; for the purpose of nulling, a higher score is given to a lower $S_{21}$ amplitude. The flowchart of the GA part is shown in Figure 10. In this program, the population is 10; the Maxgen (max generation) is 500. During the optimization procedure, the program observes the improvement. If the improvement is not significant within the last 100 generations, the program will set PM (probability of mutation) to 0.5 to prevent the program being trapped in a local optimum. This program is written using Python language, and it is based on the Pyevolve package, created by Perone [28].

3.2. The GA Procedure. To guarantee that the GA program we used is functionally correct, it is run for 5 times to 


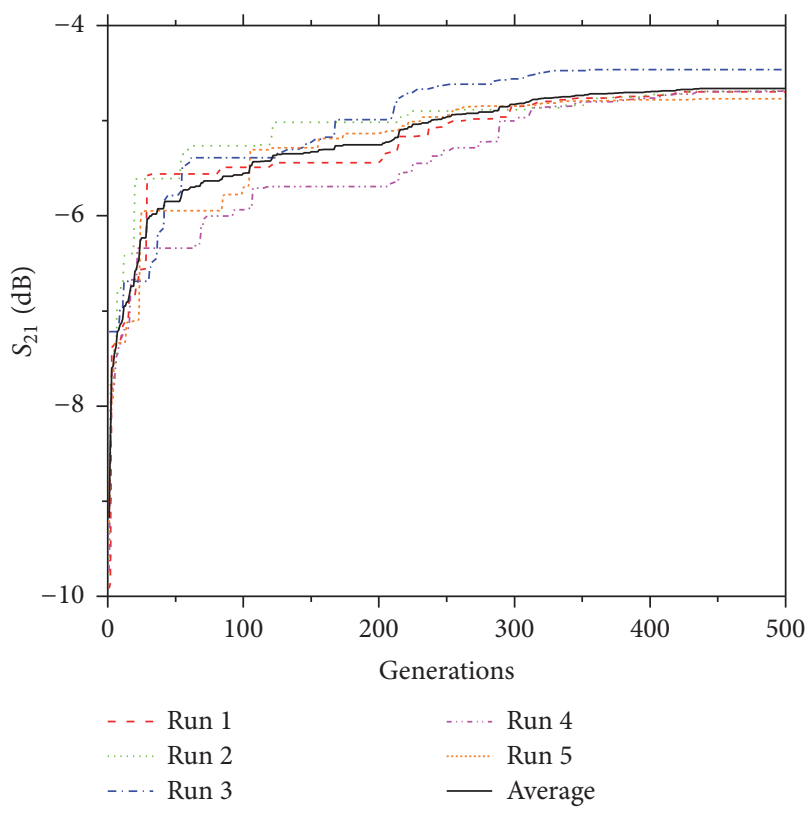

(a)

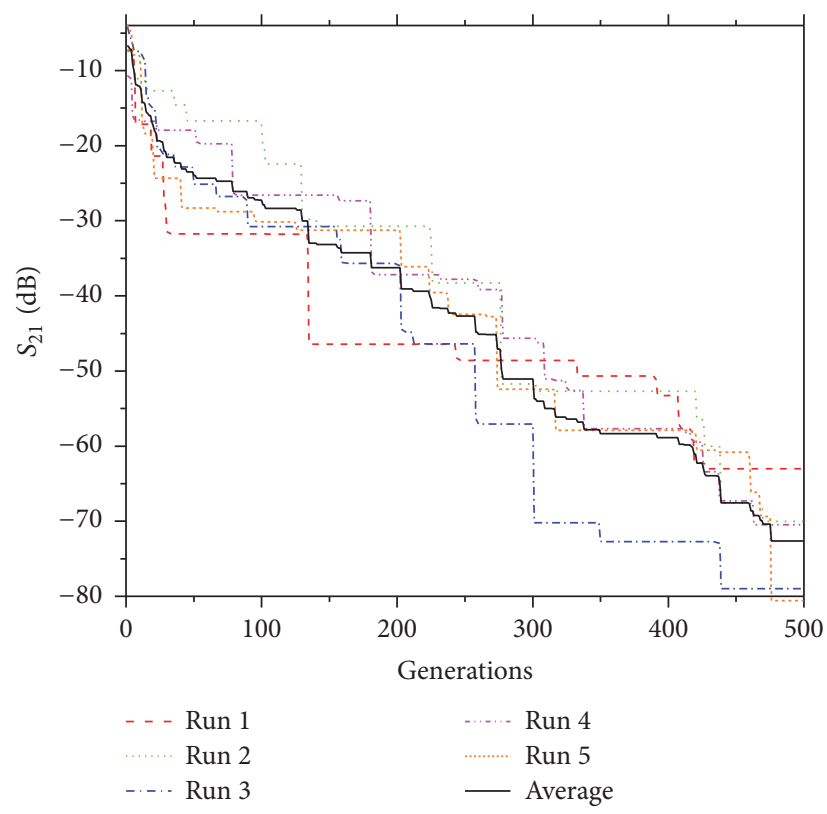

(b)

FIGURE 11: $S_{21}$ variation of the GA procedure. (a) High-gain mode. (b) Nulling mode.

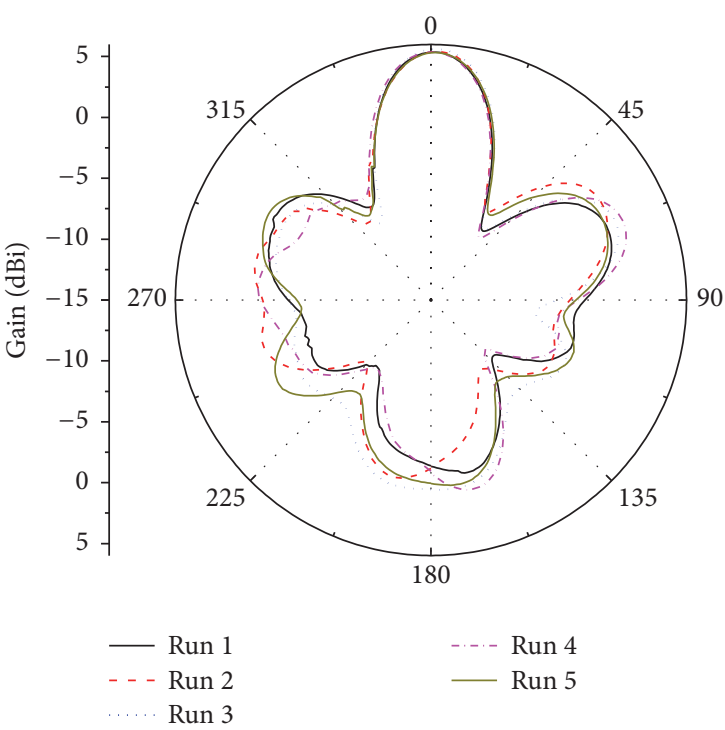

(a)

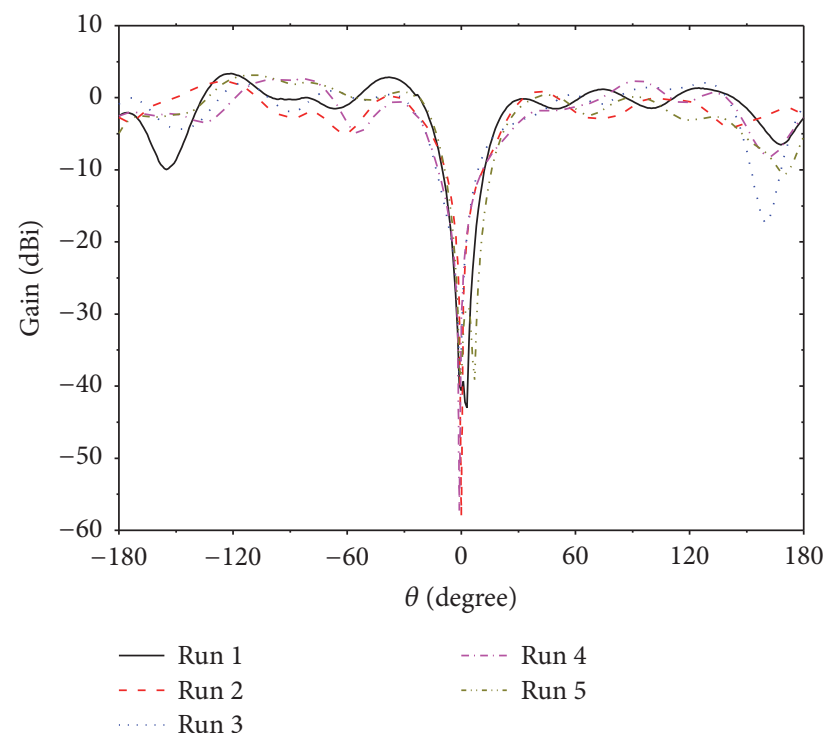

(b)

FIGURE 12: Radiation pattern for each 5 GA optimized results. (a) High-gain modes. (b) Nulling modes.

optimize the high-gain mode at $2.45 \mathrm{GHz}$. The best $S_{21}$ of each generation are stored and presented in Figure 11. It shows that the procedure is not the same for each run, but the average curve shows a more stable trend. It reaches a good result every time.

We also measured the corresponding radiation pattern of these 10 time runs, and the results are shown in Figure 12. In both cases, different trials exhibit identical behavior at the $0^{\circ}$ direction. For the 5 high-gain modes, the main lobes are almost the same, but the side lobes and backward radiation are different. The 5 nulling modes are similar at $0^{\circ}$ direction; all these 5 times can reach a deep nulling; besides this range, the radiation pattern is different for each time it runs. This is because at the other directions, there are no observation points. The GA program is not aware about the radiation of these directions. Of note, the measured nulling levels are different from the recoded ones shown in Figure 11. That is because all the environmental factors are actually considered 


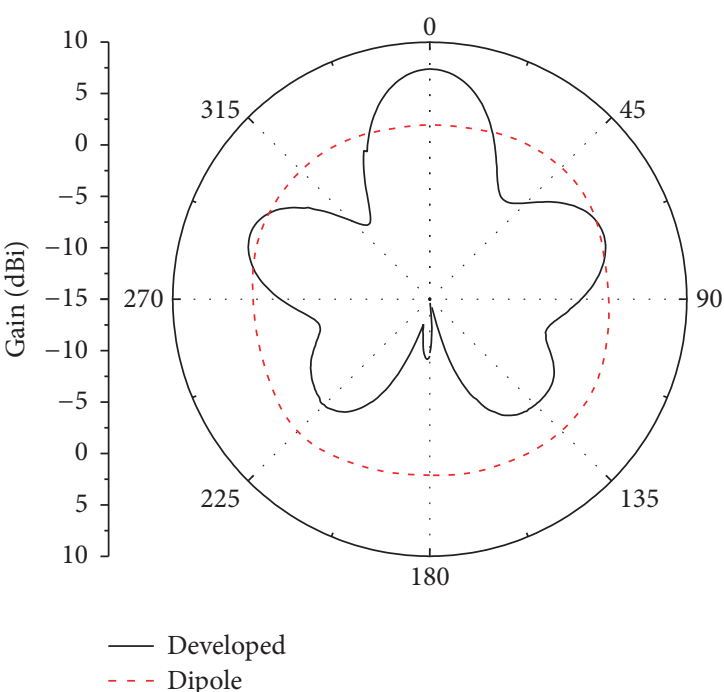

(a) $2.0 \mathrm{GHz}$

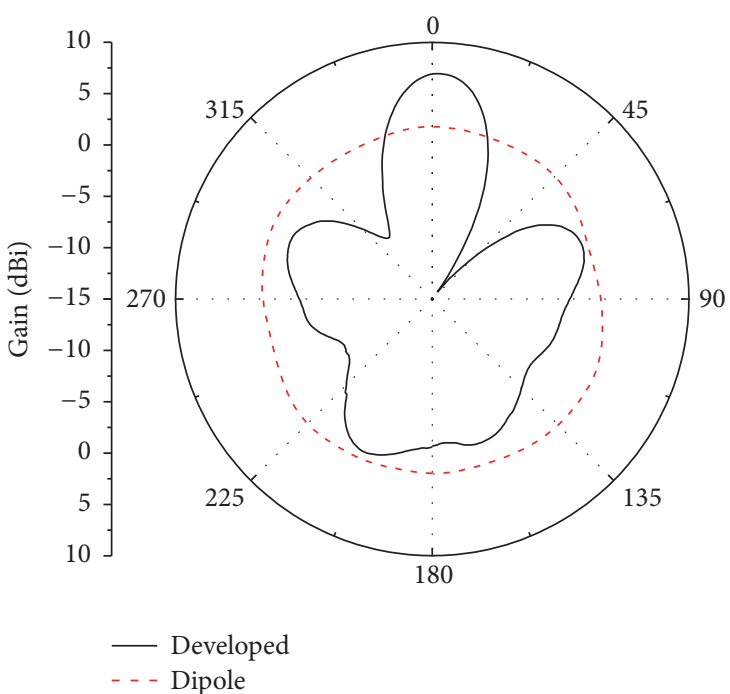

(c) $2.2 \mathrm{GHz}$

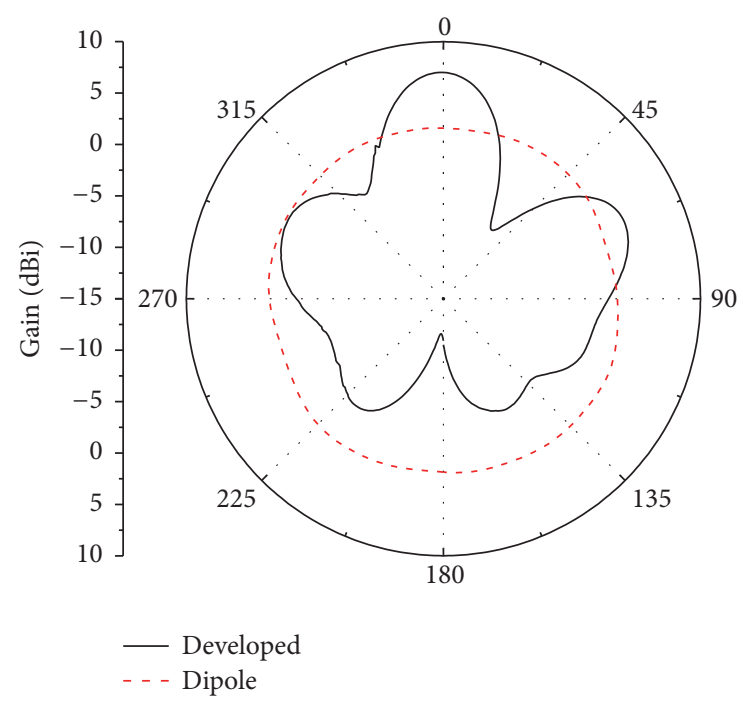

(b) $2.1 \mathrm{GHz}$

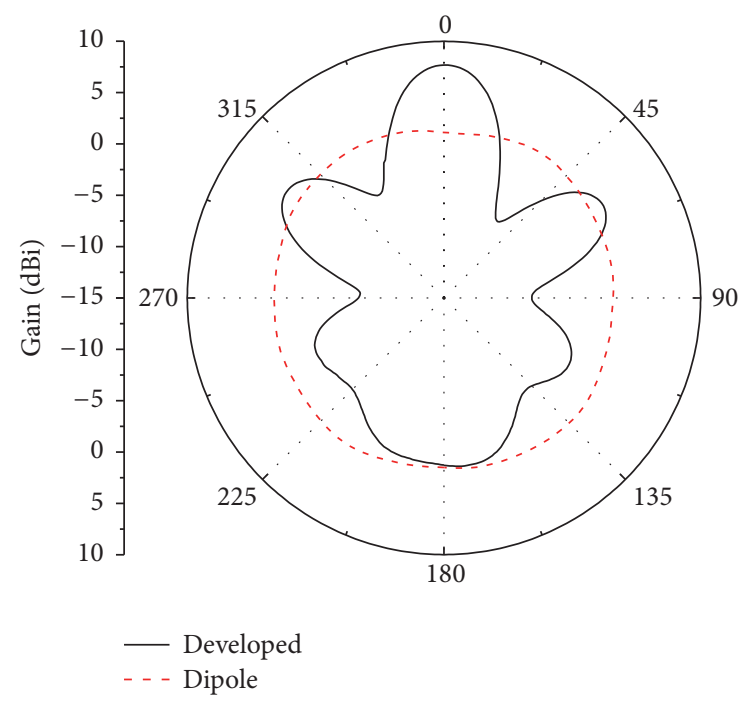

(d) $2.3 \mathrm{GHz}$

FIGURE 13: High-gain mode radiation patterns of the developed antenna from $2.0 \mathrm{GHz}$ to $2.3 \mathrm{GHz}$.

during the optimization. We need to remove the control cable to prevent it from being stretched when performing the radiation pattern measurements.

\subsection{High-Gain and Nulling Modes through the Tunable Range.} We perform the high-gain modes optimization from $2.0 \mathrm{GHz}$ to $2.7 \mathrm{GHz}$ at each $100 \mathrm{MHz}$. We use two stander dipoles to cover this spectrum: a SD2050-174 for $2.0 \mathrm{GHz}$ to $2.3 \mathrm{GHz}$ and a SD2450-181 for the other frequencies. The data of these two dipole antennas can be found on SATIMO's website. The optimized radiation patterns are shown in Figures 13 and 14 compared with the radiation pattern of the dipoles. At $0^{\circ}$ direction, which is the target direction, the gain is significantly higher than the dipoles. The data of gain, additional gain (AG) compared with the dipoles, 3 dB-beam width ( $3 \mathrm{~dB}-\mathrm{BW})$, side lobe level (SLL), and front-back ratio
(FBR) are shown in Table 1. The additional gain reaches $6.57 \mathrm{~dB}$ at $2.3 \mathrm{GHz}$, and it is higher than $5 \mathrm{~dB}$ from $2.0 \mathrm{GHz}$ to $2.4 \mathrm{GHz}$. The $3 \mathrm{~dB}$-beam width is from $26^{\circ}$ to $34^{\circ}$. It is worthwhile to point out that, the $3 \mathrm{~dB}$-beam-width in the azimuth plane obtained in this design is much smaller than those mentioned in $[5-8,10]$, the best of which achieves $47^{\circ}$. However, more efforts are being devoted to developing more smart GA-based optimization programs, for instance, a GA system that is capable of controlling all AFSS elements as independent ones (with higher degree of freedom) to achieve even higher level of gain or depression.

Another significant difference between this antenna and those mentioned in $[5-8,10]$ is that a very deep nulling level can be achieved. We alternated the goal of the GA program to achieve an increasingly lower $S_{21}$ value. The measured radiation patterns of nulling mode are shown in Figures 15 


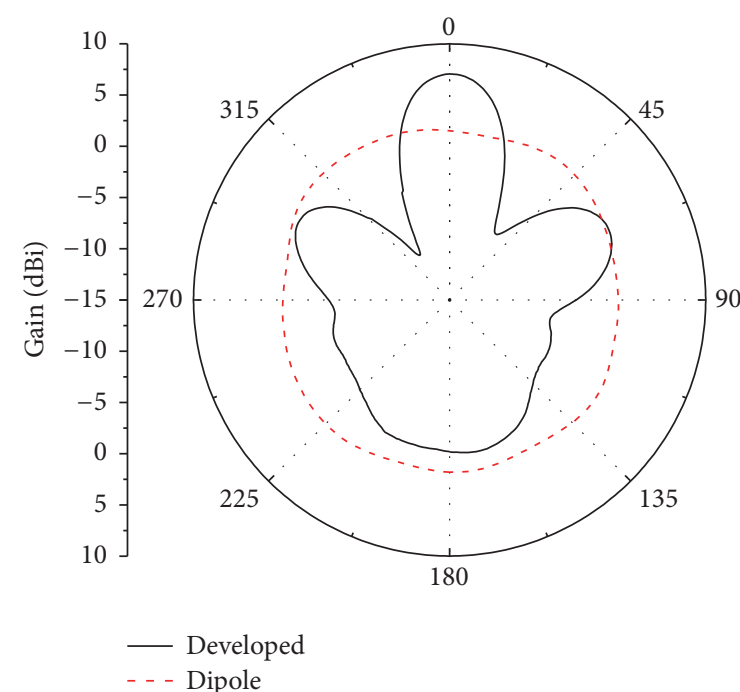

(a) $2.4 \mathrm{GHz}$

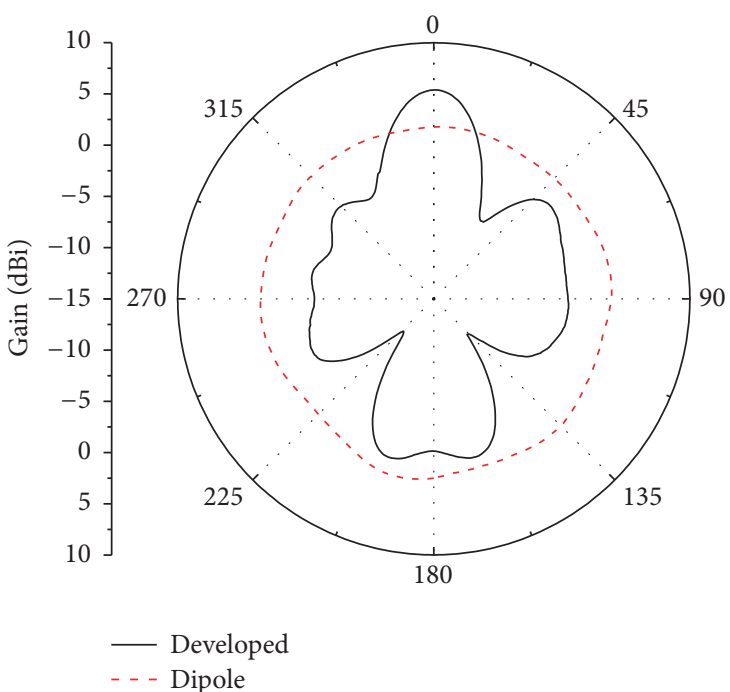

(c) $2.6 \mathrm{GHz}$

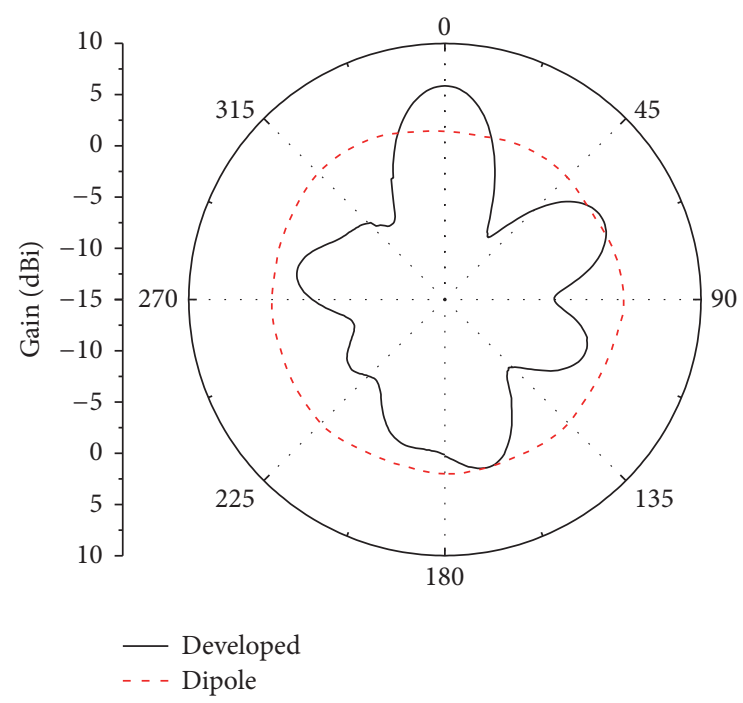

(b) $2.5 \mathrm{GHz}$

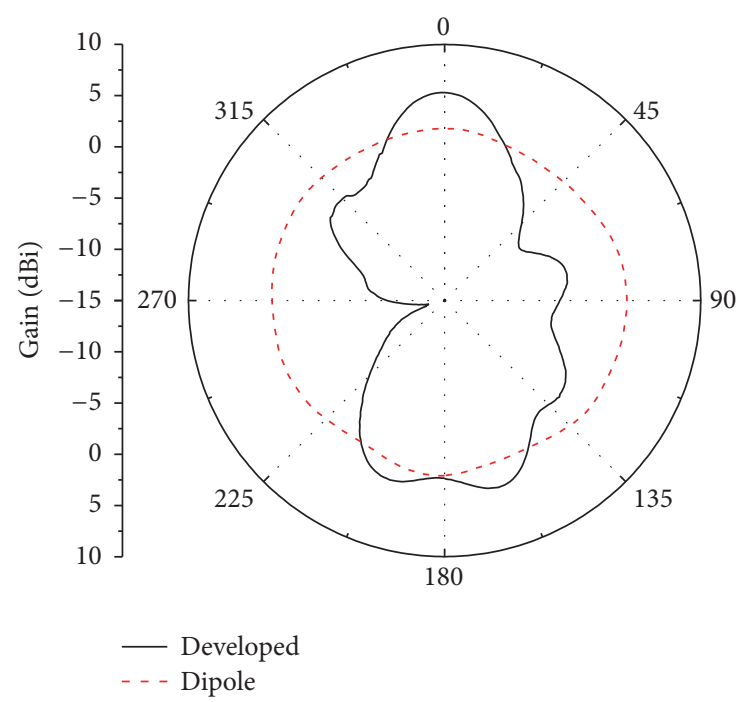

(d) $2.7 \mathrm{GHz}$

FIGURE 14: High-gain mode radiation patterns of the developed antenna from $2.4 \mathrm{GHz}$ to $2.7 \mathrm{GHz}$.

TABLE 1: Survey of the high-gain modes.

\begin{tabular}{lccccc}
\hline $\begin{array}{l}\text { Freq } \\
(\mathrm{GHz})\end{array}$ & $\begin{array}{c}\text { Gain } \\
(\mathrm{dBi})\end{array}$ & AG $(\mathrm{dB})$ & $\begin{array}{c}3 \mathrm{~dB}-\mathrm{BW} \\
(\text { degree })\end{array}$ & SLL $(\mathrm{dBi})$ & FBR $(\mathrm{dB})$ \\
\hline 2.0 & 7.37 & 5.42 & 31 & 3.54 & 16.84 \\
2.1 & 6.99 & 5.40 & 30 & 4.10 & 17.9 \\
2.2 & 6.96 & 5.14 & 26 & 0.50 & 7.56 \\
2.3 & 7.69 & 6.57 & 27 & 3.49 & 6.43 \\
2.4 & 7.05 & 5.53 & 27 & 1.98 & 7.26 \\
2.5 & 5.86 & 4.44 & 27 & 2.27 & 5.70 \\
2.6 & 5.39 & 3.61 & 26 & -0.45 & 5.55 \\
2.7 & 5.30 & 3.53 & 34 & Null & 2.96 \\
\hline
\end{tabular}

and 16. Here, we use Cartesian coordinate and the $x$-axis is transferred from $0^{\circ}-360^{\circ}$ to $\pm 180^{\circ}$ for a clearer look. At the desired $0^{\circ}$, the nulling levels are below $-30 \mathrm{dBi}$ and even reach $-56.41 \mathrm{dBi}$ at $2.7 \mathrm{GHz}$. These results show that the proposed antenna can be used for noise cancelling and angle finding applications.

The antenna matching is influenced by the AFSS and the status. The measured $S_{11}$ of 8 high-gain and 8 nulling modes are shown in Figure 17, compared with the dipoles' original $S_{11}$. For both of the dipoles, $S_{11}$ are influenced by the AFSS and the working status. The results are still acceptable, since all the curves are around $-10 \mathrm{~dB}$. Moreover, it is obvious from Figure 17 that the $S_{11}$ value of high-gain mode optimized for target frequency does not have a best value at each frequency; this is due to the reason that the optimization process is dominated by $S_{21}$ performance and therefore makes sacrifice for the efficiency. However, this issue is still worthy of a further research to achieve a better matching performance, and more efforts are being devoted to 


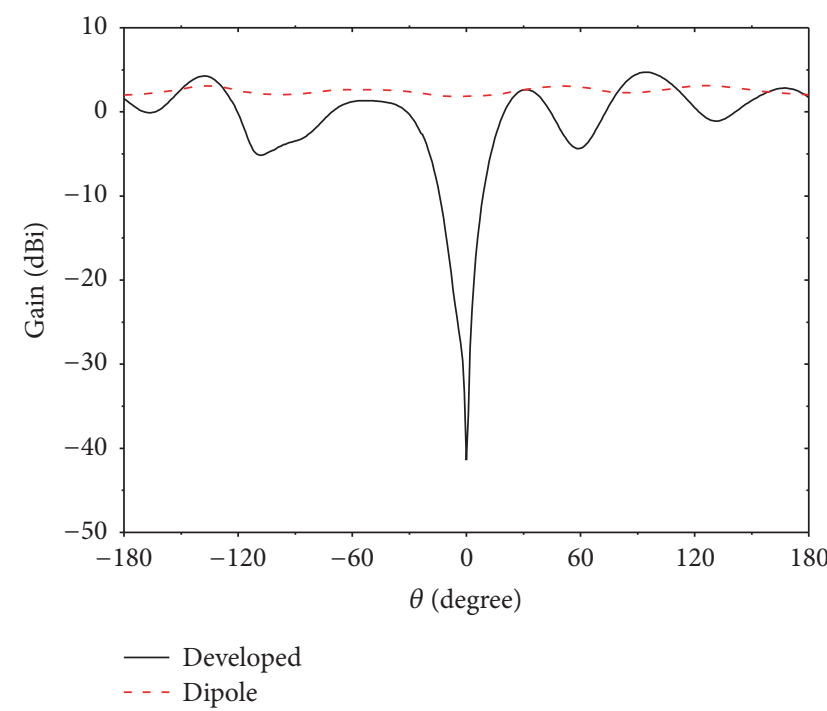

(a) $2.0 \mathrm{GHz}$

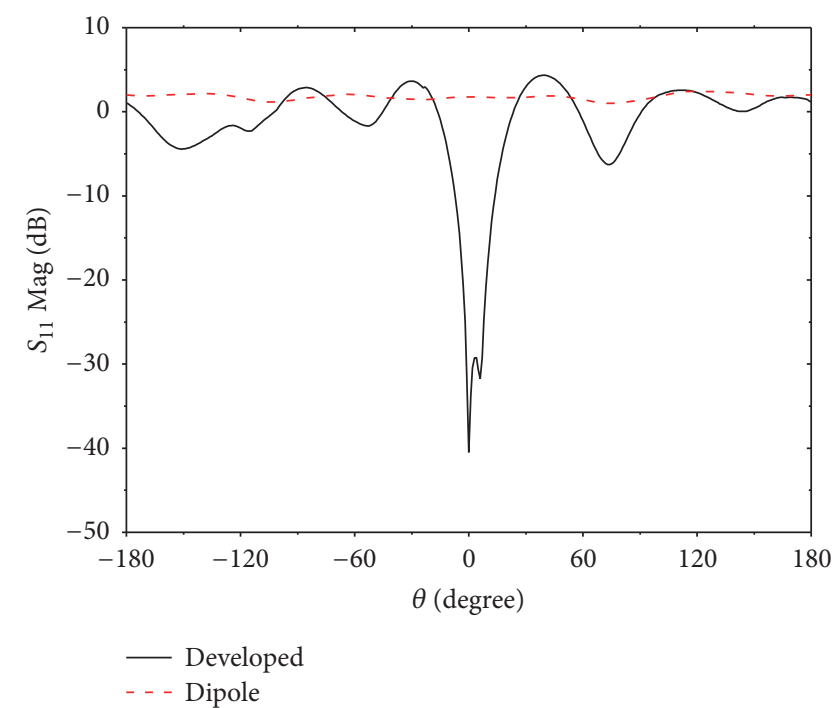

(c) $2.2 \mathrm{GHz}$

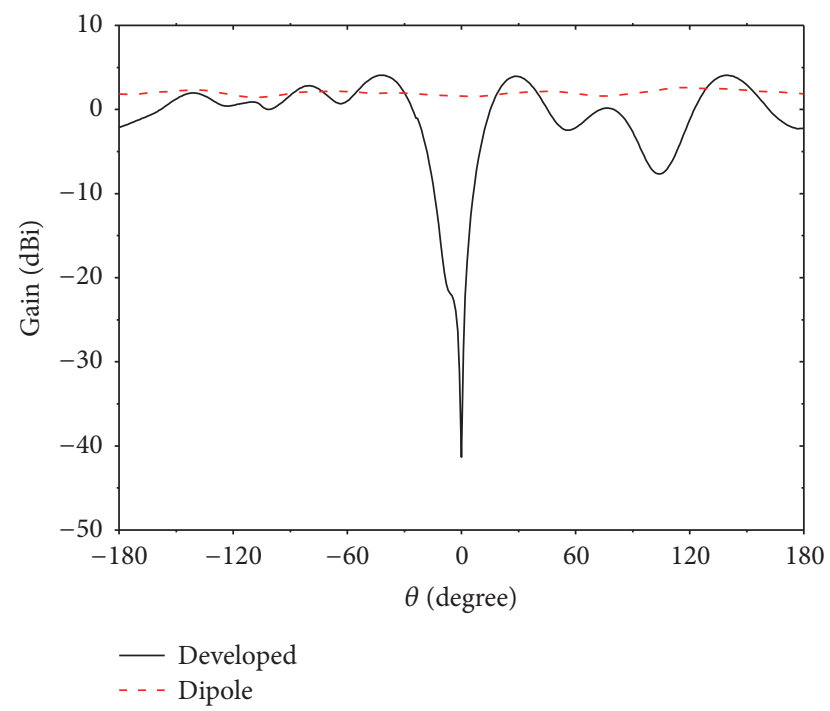

(b) $2.1 \mathrm{GHz}$

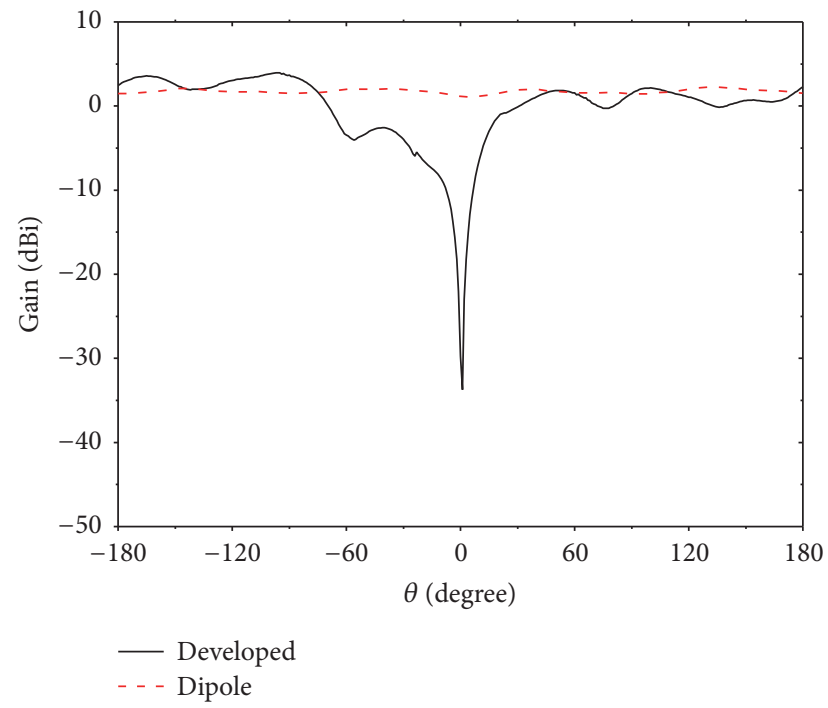

(d) $2.3 \mathrm{GHz}$

FIGURE 15: Nulling mode radiation patterns of the developed antenna from $2.0 \mathrm{GHz}$ to $2.3 \mathrm{GHz}$.

developing independently controlled AFSS units and smart optimization algorithms, which would help improve such behavior significantly.

3.4. Continuously Steerable Ability. Another benefit of this antenna is that it is continuously tunable compared with the on/off method based electronically steerable antennas. The proposed antenna contains 16 AFSS columns and is perfectly central symmetric. Thus, there are 32 axes of symmetry. Therefore, we only need to prove that the antenna is continuously tunable within each $11.25^{\circ}$ sector. To verify this ability, we shift the target direction from $0^{\circ}$ to $11.25^{\circ}$ by $3.75^{\circ}$ to optimize the high-gain mode. Because for the purpose of communication, where high-gain mode is mostly employed, a $3.75^{\circ}$ tunability is quite enough for a main lobe of $26^{\circ}$ to $34^{\circ}$ width. For the nulling mode, it is usually used for strong noise isolation and angle finding purpose, which often requires an accurate tunability; we shift the target direction for each $1^{\circ}$ from $0^{\circ}$ to $11^{\circ}$. The results are shown in Figure 18. For the 4 high-gain modes, the measured gains are $5.33 \mathrm{dBi}$, $5.17 \mathrm{dBi}, 5.71 \mathrm{dBi}$, and $5.31 \mathrm{dBi}$, and they point to $0^{\circ}, 7^{\circ}, 9^{\circ}$, and $11^{\circ}$, respectively. The directions have some error, but the continuous tunability is basically approved. For the nulling modes, the nulling angles point to each $1^{\circ}$ precisely.

3.5. Beam Bandwidth Investigation. Another important parameter for electronically steerable antenna is the beam bandwidth. For instance, to use this antenna for a $2.4 \mathrm{GHz}$ WiFi system, there are two bandwidths we should be concerned about. One is $2.4-2.483 \mathrm{GHz}$, which is the total 


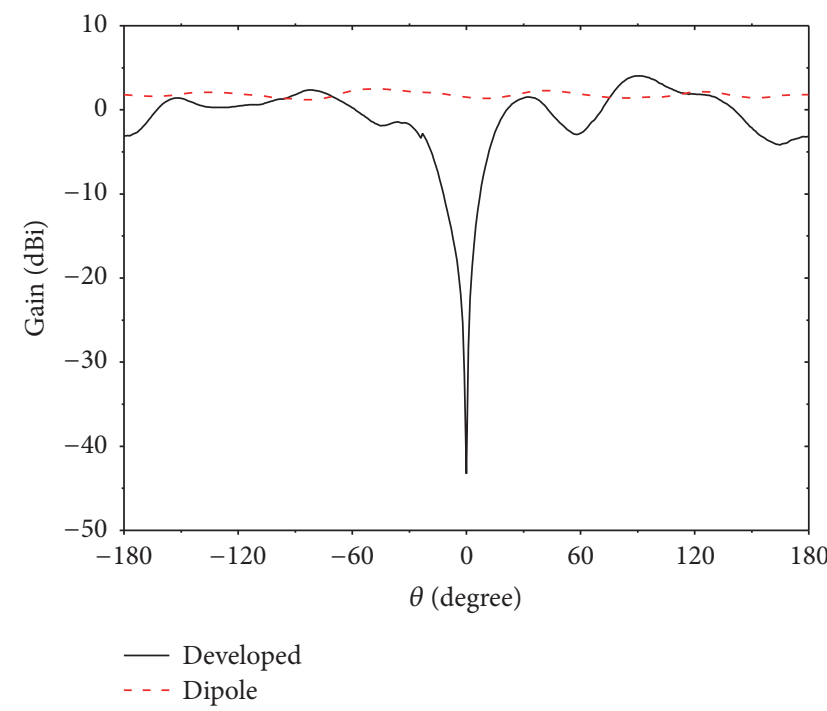

(a) $2.4 \mathrm{GHz}$

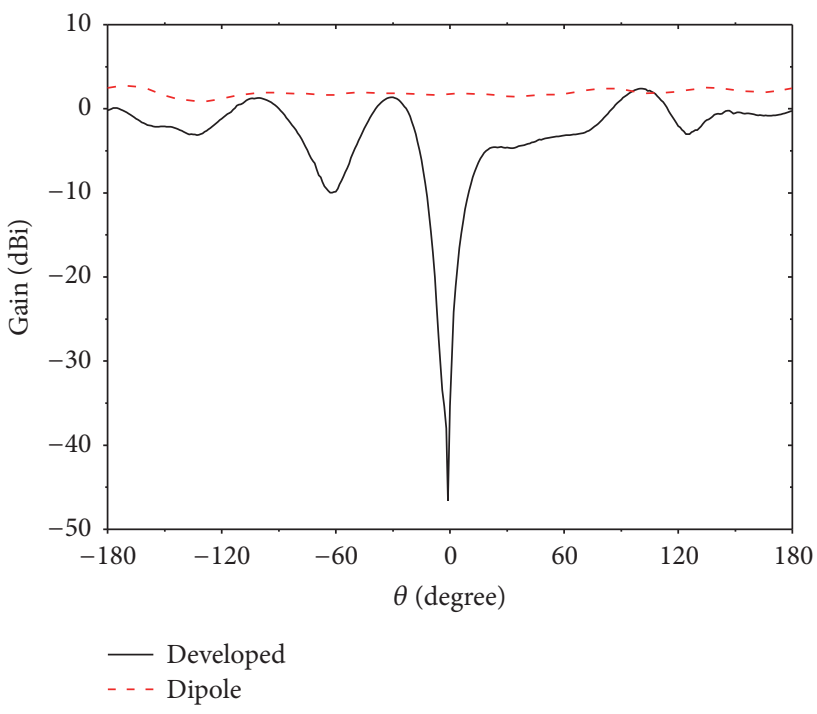

(c) $2.6 \mathrm{GHz}$

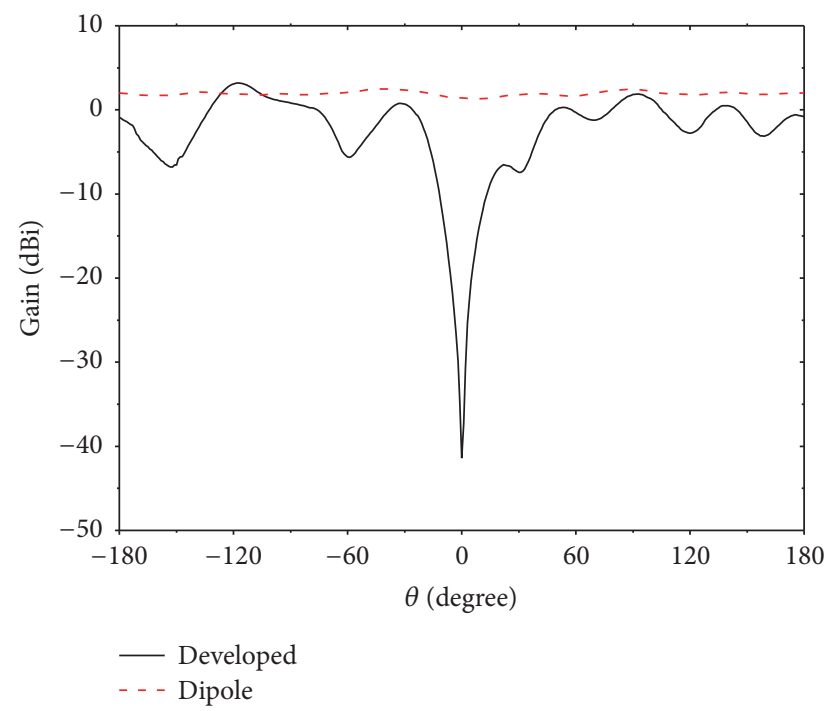

(b) $2.5 \mathrm{GHz}$

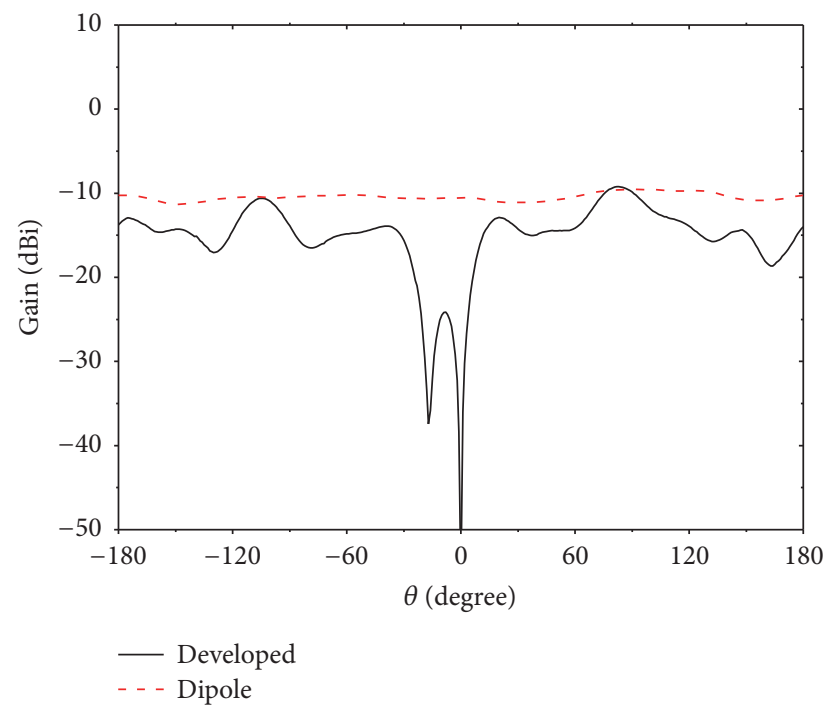

(d) $2.7 \mathrm{GHz}$

FIGURE 16: Nulling mode radiation patterns of the developed antenna from $2.4 \mathrm{GHz}$ to $2.7 \mathrm{GHz}$.

2.4 GHz WiFi; the other bandwidth is the channel bandwidth, which is $22 \mathrm{MHz}$ for IEEE $802.11 \mathrm{~b}, 20-16 \mathrm{MHz}$ for $802.11 \mathrm{~g} / \mathrm{n}$ and some other standards. If the antenna control method is enclosed within the protocol, the bandwidth of the beam only needs to remain invariant in the channel bandwidth. Once the system changes the working channel, the antenna can change correspondingly. If the antenna control method is not included in the protocol, beam bandwidth has to cover the whole spectrum of this protocol. This is especially important for a frequency-hopping spread spectrum (FHSS) system such as the well-known Bluetooth system. In this work, the antenna is supposed to be narrow band. That is, because unlike attenuators and phase shifters based steerable antennas, the AFSS cannot maintain the same amplitude and phase value in a wide band. So, it is necessary to find out the beam bandwidth performance. We optimized the gain at $2.44 \mathrm{GHz}$ and measured radiation pattern from 2.40 to $2.49 \mathrm{GHz}$ by each $10 \mathrm{MHz}$. The results are shown in Figure 19(a). The gains are from $7.02 \mathrm{dBi}$ to $5.53 \mathrm{dBi}$, which means that when using this antenna for a WiFi system without optimization to each channel or for a Bluetooth system, there will be $1.49 \mathrm{~dB}$ difference for different channels. It is no big problem, but we would like to see if this issue can be improved. To do so, we modified the program to observe the $S_{21}$ of these 10 frequency points and scored the $S_{21}$ with an additional condition to constrain the mean square error of the 10 amplitudes of $S_{21}$ to be smaller than 0.2. The responding radiation patterns are shown in Figure 19(b). The difference of gain is smaller.

For a similar reason we performed a similar method to learn the beam bandwidth of the nulling mode. We set the average of these 10 amplitudes of $S_{21}$ as the target instead of 


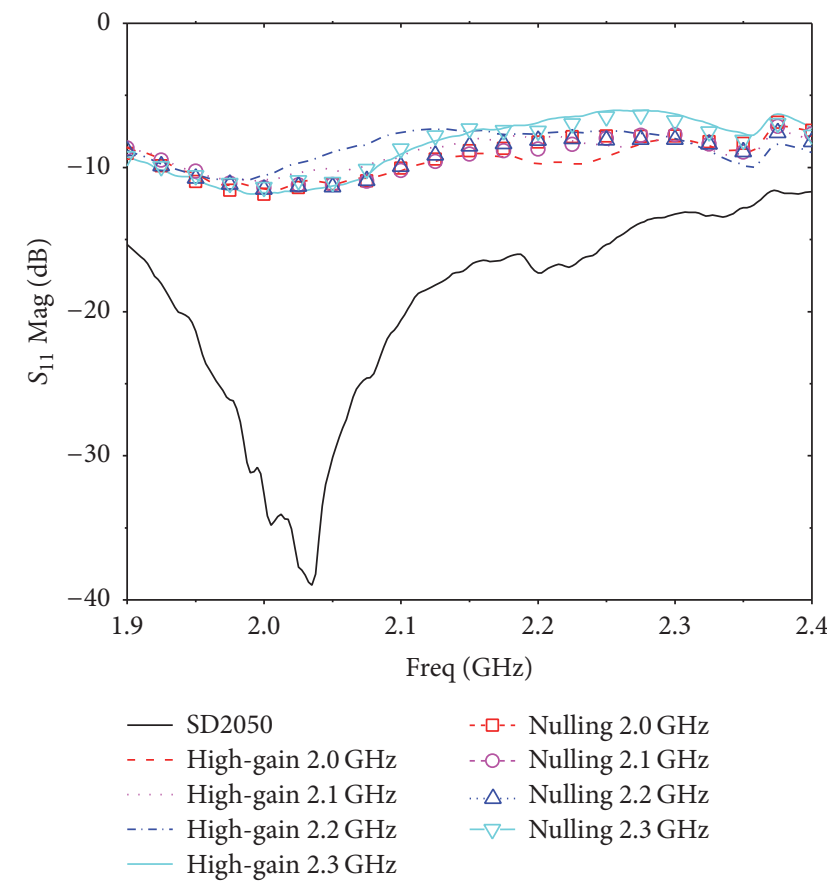

(a)

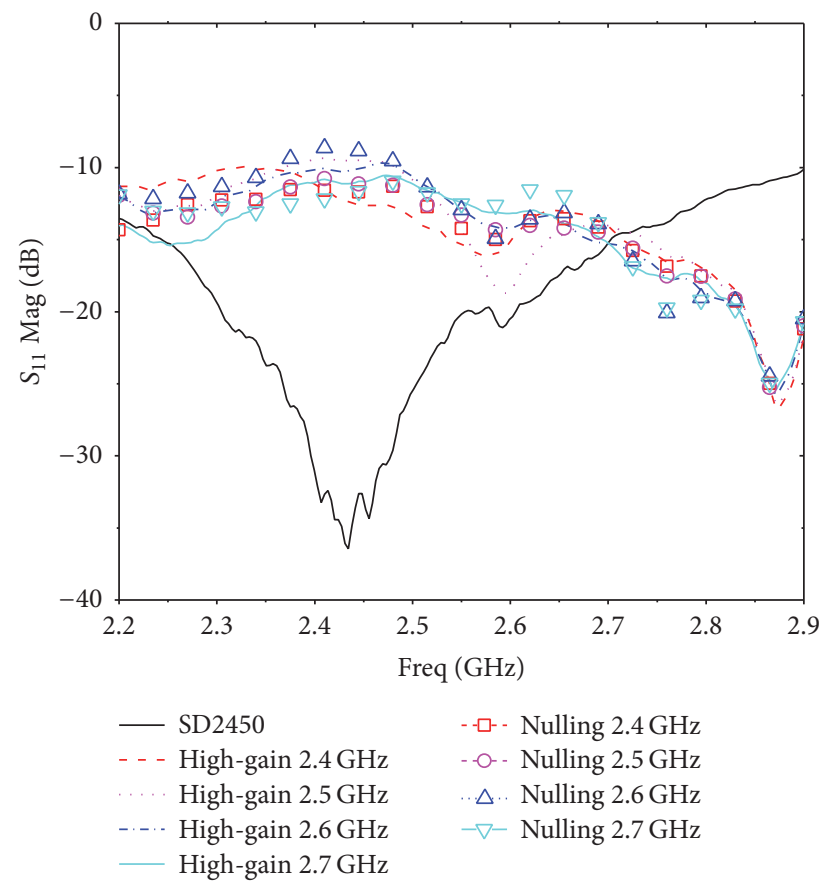

(b)

Figure 17: The influence on $S_{11}$. (a) SD2050 and 8 modes. (b) SD2450 and 8 modes.

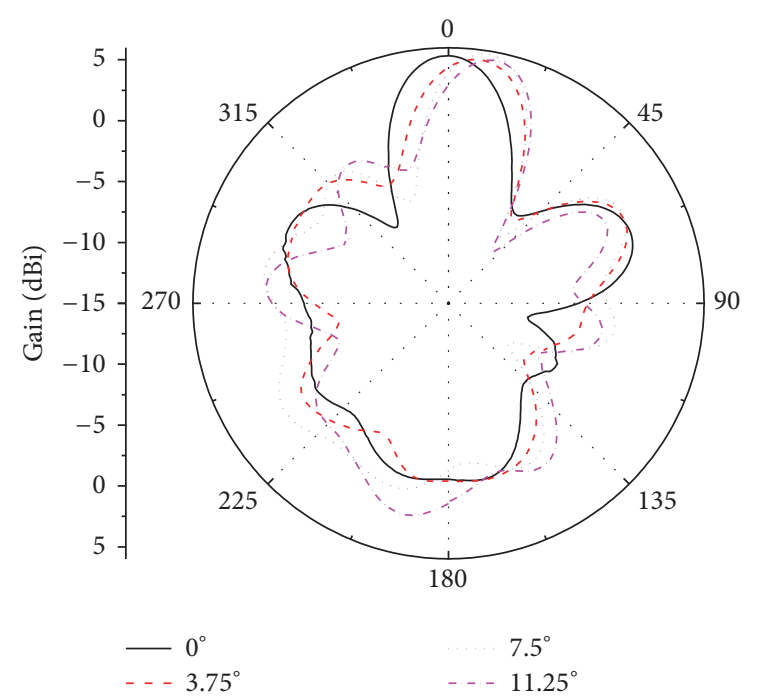

(a)
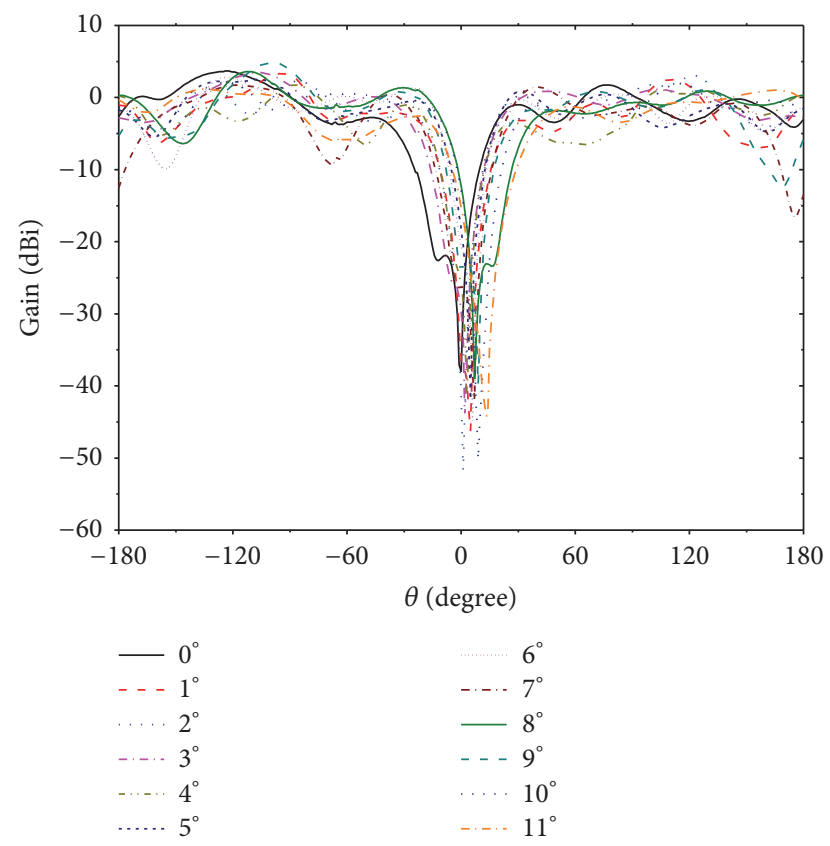

(b)

FIGURE 18: Radiation patterns for examining the continuous tunability. (a) High-gain modes. (b) Nulling modes. 


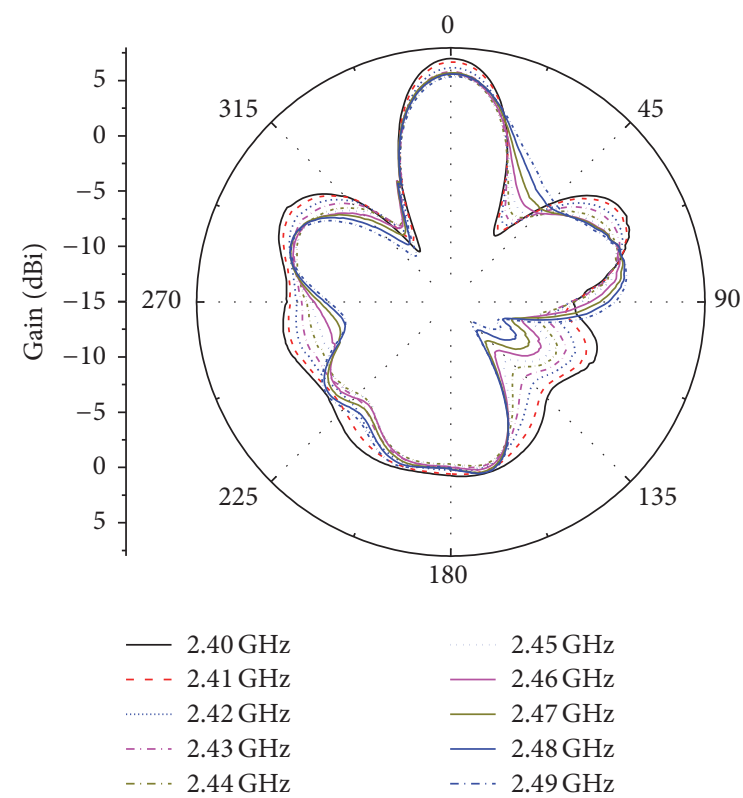

(a)

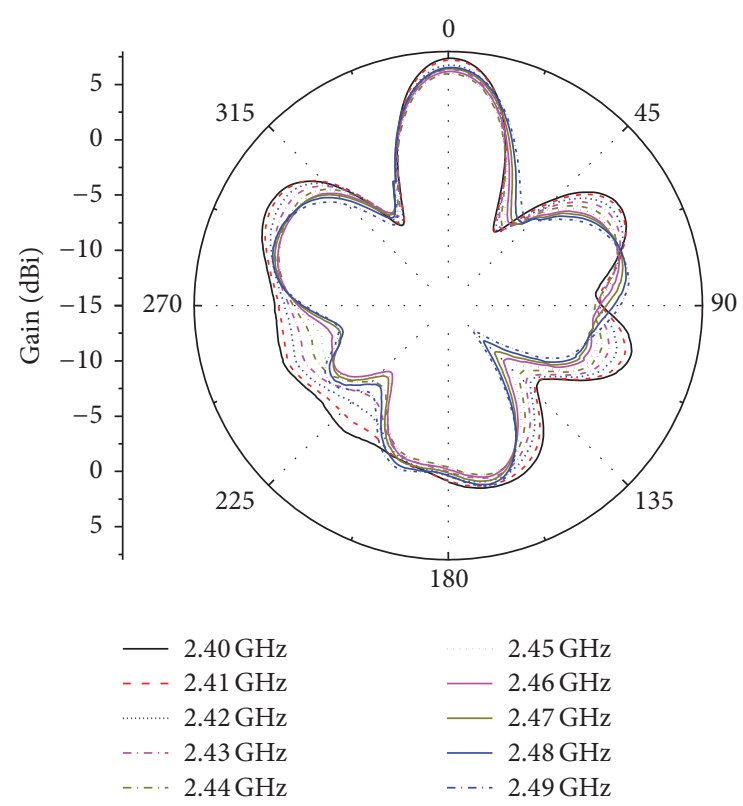

(b)

FIGURE 19: High-gain mode radiation patterns of WiFi band for the examination of beam bandwidth. (a) Without constraint. (b) Constrained by mean square error condition.

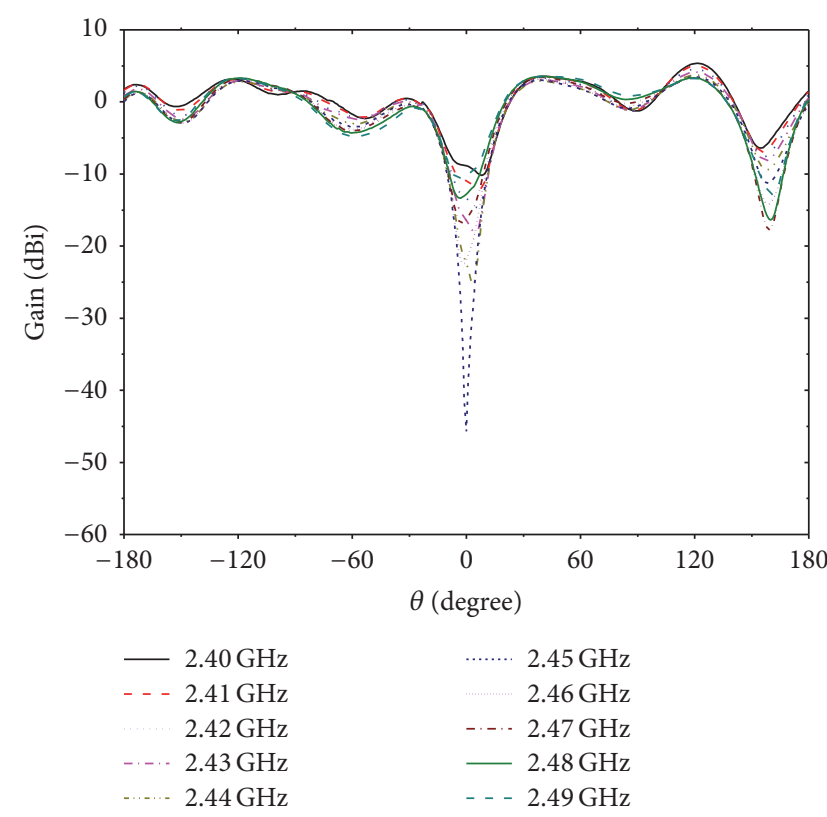

(a)

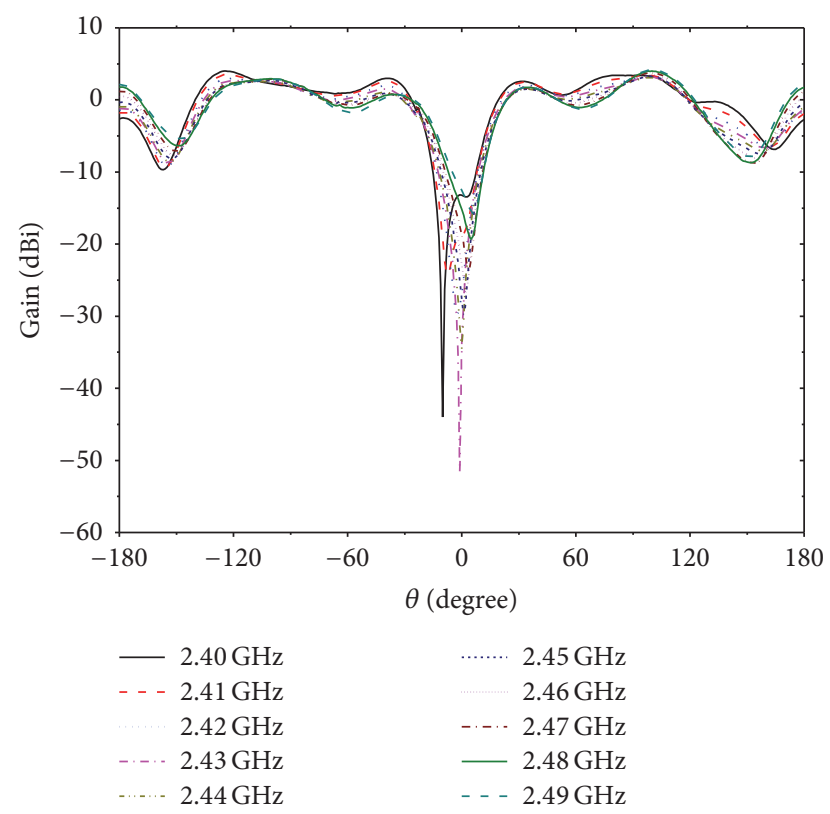

(b)

FIGURE 20: Nulling mode radiation patterns of WiFi band for the examination of beam bandwidth. (a) Results of single frequency goal. (b) Results of multiple frequency average goal.

the mean square error. The results are shown in Figure 20. The improvement is significant. For most frequencies, the nulling levels are below $-20 \mathrm{dBi}$.

\section{Conclusion}

In this paper, a highly agile electronically steerable active smart dome antenna is proposed. This antenna takes advantage of the amplitude and phase response of the employed AFSS, as well as a self-made genetic algorithm based optimization system to experimentally realize high-gain modes and nulling modes within the frequency tunable range of $2.0 \mathrm{GHz}$ to $2.7 \mathrm{GHz}$. The antenna is continuously steerable and outperforms many PIN-diode based FSS configurations in agility. The measured results achieved additional gain of up to $6.57 \mathrm{~dB}$ and a deep nulling level of $-56 \mathrm{dBi}$. For the high-gain 
modes, the $-3 \mathrm{~dB}$ beam widths are $26^{\circ}-34^{\circ}$, while for the nulling mode, they can offer a steering resolution of $1^{\circ}$. Beam bandwidth measurement shows that it can offer stable highgain mode and nulling mode for $2.4 \mathrm{GHz}$ WiFi band.

\section{Competing Interests}

The authors declare that they have no competing interests.

\section{Acknowledgments}

This work was supported by the National Natural Science Foundation of China (61601391) and Educational Commission of Fujian Province, China (JZ160449).

\section{References}

[1] H. Krim and M. Viberg, "Two decades of array signal processing research: the parametric approach," IEEE Signal Processing Magazine, vol. 13, no. 4, pp. 67-94, 1996.

[2] A. Alexiou and M. Haardt, "Smart antenna technologies for future wireless systems: trends and challenges," IEEE Communications Magazine, vol. 42, no. 9, pp. 90-97, 2004.

[3] S. Farzaneh and A.-R. Sebak, "A novel amplitude-phase weighting for analog microwave beamforming," IEEE Transactions on Antennas and Propagation, vol. 54, no. 7, pp. 1997-2008, 2006.

[4] J. Kennedy and M. C. Sullivan, "Direction finding and "smart antennas" using software radio architectures," IEEE Communications Magazine, vol. 33, no. 5, pp. 62-68, 1995.

[5] M. Roig, M. Sazegar, Y. Zheng, and R. Jakoby, "Tunable frequency selective surface based on ferroelectric ceramics for beam steering antennas," in Proceedings of the 7th German Microwave Conference (GeMiC '12), pp. 1-4, March 2012.

[6] A. Edalati and T. A. Denidni, "High-gain reconfigurable sectoral antenna using an active cylindrical fss structure," IEEE Transactions on Antennas and Propagation, vol. 59, no. 7, pp. 2464-2472, 2011.

[7] M. N. Jazi and T. A. Denidni, "Agile radiation-pattern antenna based on active cylindrical frequency selective surfaces," IEEE Antennas and Wireless Propagation Letters, vol. 9, pp. 387-388, 2010.

[8] M. Niroo-Jazi and T. A. Denidni, "Electronically sweepingbeam antenna using a new cylindrical frequency-selective surface," IEEE Transactions on Antennas and Propagation, vol. 61, no. 2, pp. 666-676, 2013.

[9] L. Zhang, Q. Wu, and T. A. Denidni, "Electronically radiation pattern steerable antennas using active frequency selective surfaces," IEEE Transactions on Antennas and Propagation, vol. 61, no. 12, pp. 6000-6007, 2013.

[10] B. Liang, B. Sanz-Izquierdo, E. A. Parker, and J. C. Batchelor, "Cylindrical slot FSS configuration for beam-switching applications," IEEE Transactions on Antennas and Propagation, vol. 63, no. 1, pp. 166-173, 2015.

[11] W. Pan, C. Huang, P. Chen, M. Pu, X. Ma, and X. Luo, "A beam steering horn antenna using active frequency selective surface," IEEE Transactions on Antennas and Propagation, vol. 61, no. 12, pp. 6218-6223, 2013.

[12] L. C. Godara, Smart Antennas, CRC Press, 2014.

[13] D. Schurig, J. J. Mock, and D. R. Smith, "Electric-field-coupled resonators for negative permittivity metamaterials," Applied Physics Letters, vol. 88, no. 4, Article ID 041109, 2006.
[14] W. Withayachumnankul, C. Fumeaux, and D. Abbott, "Planar array of electric-LC resonators with broadband tunability," IEEE Antennas and Wireless Propagation Letters, vol. 10, pp. 577-580, 2011.

[15] J. Y. Kim, D. S. Kim, D. W. Woo, D. S. Shin, W. S. Park, and W. Hwang, "Electromagnetic wave characteristics of secondorder composite frequency-selective surfaces fabricated by ebeam evaporator," Electronics Letters, vol. 50, no. 3, pp. 189-190, 2014.

[16] J. M. Zendejas, J. P. Gianvittorio, Y. Rahmat-Samii, and J. W. Judy, "Magnetic MEMS reconfigurable frequency-selective surfaces," Journal of Microelectromechanical Systems, vol. 15, no. 3, pp. 613-623, 2006.

[17] B. Schoenlinner, A. Abbaspour-Tamijani, L. C. Kempel, and G. M. Rebeiz, "Switchable low-loss RF MEMS Ka-band frequencyselective surface," IEEE Transactions on Microwave Theory and Techniques, vol. 52, no. 11, pp. 2474-2481, 2004.

[18] J. P. Gianvittorio, J. Zendejas, Y. Rahmat-Samii, and J. Judy, "Reconfigurable MEMS-enabled frequency selective surfaces," Electronics Letters, vol. 38, no. 25, pp. 1627-1628, 2002.

[19] I. J. Gupta and A. A. Ksienski, "Effect of mutual coupling on the performance of adaptive arrays," IEEE Transactions on Antennas and Propagation, vol. 31, no. 5, pp. 785-791, 1983.

[20] K. S. Rao, "On the collimation phase error computation of a space-fed planar phased array," IEEE Transactions on Antennas and Propagation, vol. 31, no. 1, pp. 154-156, 1983.

[21] C. H. Hightower, S. H. Wong, A. R. Perkons, and C. I. Igwe, "A space-fed phased array for surveillance from space," IEEE Aerospace and Electronic Systems Magazine, vol. 6, no. 5, pp. 1317, 1991.

[22] M. Stoneback, M. Stoneback, and Y. Kuga, "Feasibility study of a wirelessly controlled and powered space-fed phased array antenna," IEEE Transactions on Antennas and Propagation, vol. 61, no. 12, pp. 5984-5991, 2013.

[23] E. Jacobs, "Phase error effects on gain and sidelobe level of a space-fed array," IEEE Transactions on Antennas and Propagation, vol. 28, no. 2, pp. 243-246, 1980.

[24] S. W. Schneider and B. A. Munk, "The scattering properties of "super dense" arrays of dipoles," IEEE Transactions on Antennas and Propagation, vol. 42, no. 4, pp. 463-472, 1994.

[25] R. L. Haupt, “Thinned arrays using genetic algorithms," IEEE Transactions on Antennas and Propagation, vol. 42, no. 7, pp. 993-999, 1994.

[26] F. J. Ares-Pena, J. A. Rodriguez-Gonzalez, E. Villanueva-Lopez, and S. R. Rengarajan, "Genetic algorithms in the design and optimization of antenna array patterns," IEEE Transactions on Antennas and Propagation, vol. 47, no. 3, pp. 506-510, 1999.

[27] K.-K. Yan and Y. Lu, "Sidelobe reduction in array-pattern synthesis using genetic algorithm," IEEE Transactions on Antennas and Propagation, vol. 45, no. 7, pp. 1117-1122, 1997.

[28] C. S. Perone, Pyevolve project, http://pyevolve.sourceforge.net/. 


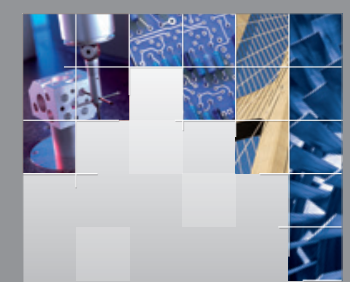

\section{Enfincering}
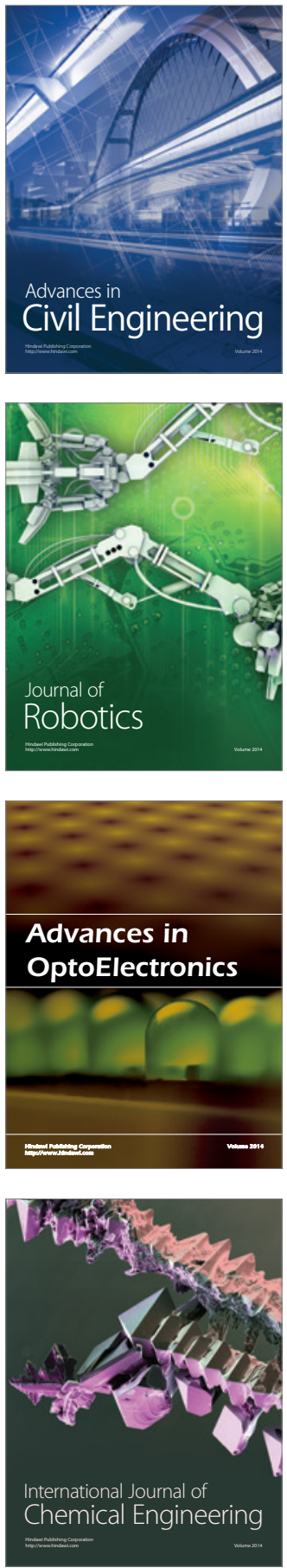

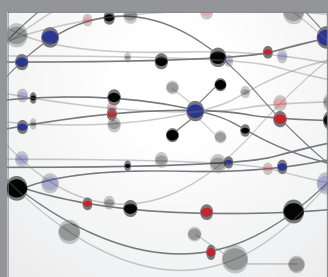

The Scientific World Journal

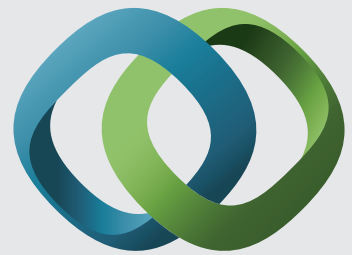

\section{Hindawi}

Submit your manuscripts at

https://www.hindawi.com
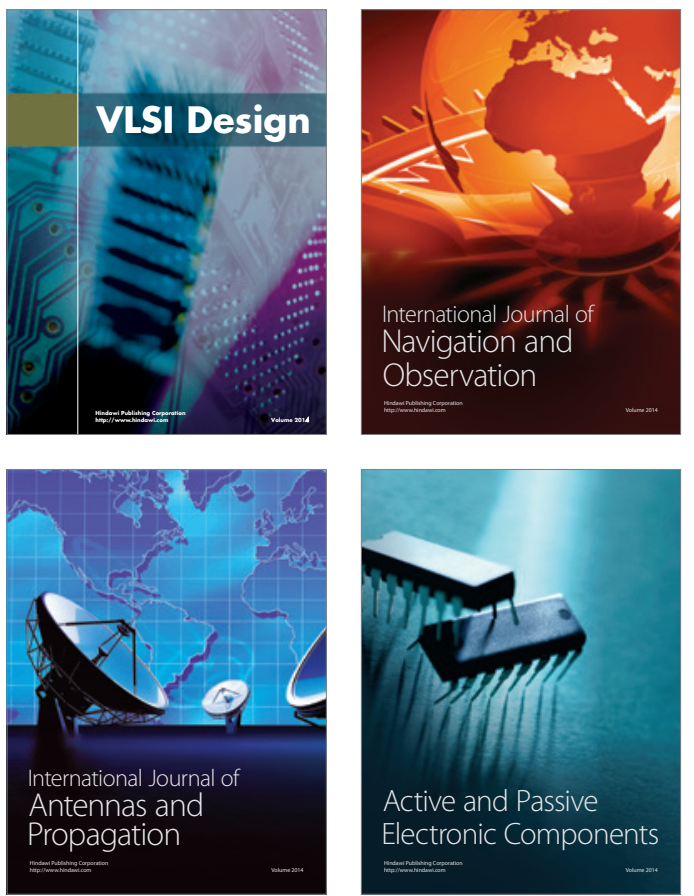
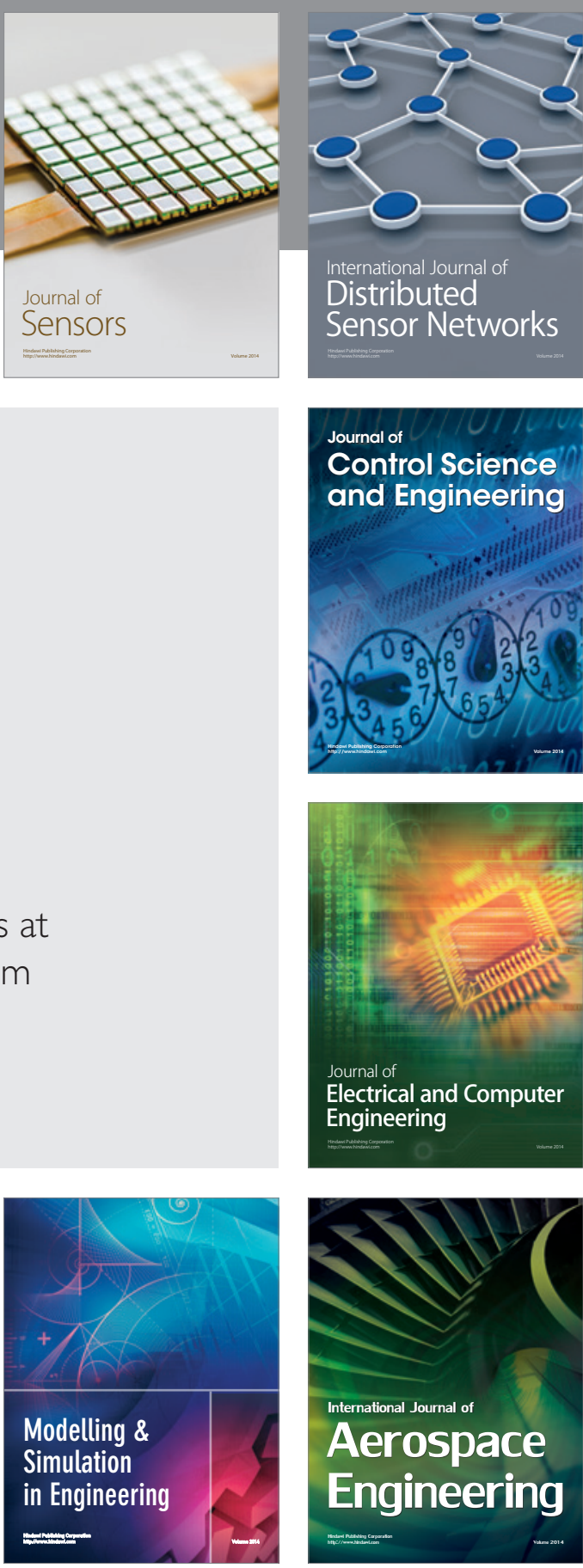

International Journal of

Distributed

Sensor Networks

$-$

Joumal of

Control Science

and Engineering
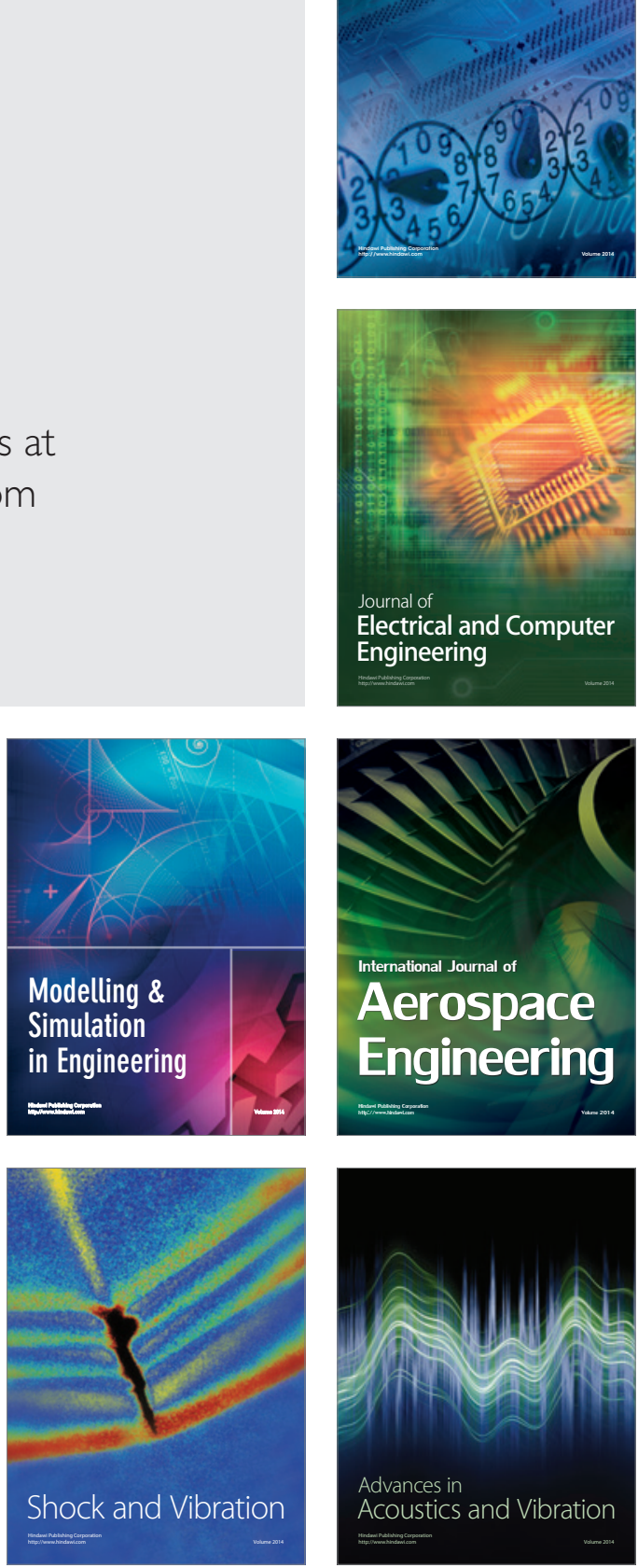